\title{
Accessing Monosubstituted Cyclopentadienyl Rhodium(I) and Iridium(I) Complexes by a Simultaneous Nucleophilic Addition-Metalation Approach to Fulvenes
}

\author{
Aragorn Laverny and Nicolai Cramer* \\ Laboratory of Asymmetric Catalysis and Synthesis, Institute of Chemical Sciences and \\ Engineering, Ecole Polytechnique Fédérale de Lausanne (EPFL), SB-ISIC, BCH4305, \\ 1015 Lausanne, Switzerland \\ *e-mail: nicolai.cramer@epfl.ch
}

Supplementary Information

Table of Content:

General Methods $\quad$ S2

Rhodium and Iridium Precursors Synthesis $\quad S 4$

Fulvene Synthesis $\quad S 4$

Crystallographic Data $\quad S 7$

References $\quad S 8$

$\begin{array}{ll}N M R \text {-Spectra } & \text { S9 }\end{array}$ 


\section{General Methods}

\section{Experimental Procedures, Reagents and Glassware}

All reactions were carried out under air atmosphere without any precaution for moisture, unless otherwise indicated. Reagents and solvents were purchased from Aldrich, Acros, Alfa Aesar, Abcr, or $\mathrm{TCl}$ and used as obtained from the suppliers. Dry diethyl ether $\left(\mathrm{Et}_{2} \mathrm{O}\right)$, dichloromethane (DCM), toluene and tetrahydrofuran (THF) were purified by an Innovative Technology Solvent Delivery System. Ethyl acetate (AcOEt), dichloromethane and pentane for filtration, transfer, chromatography, and recrystallization were purchased from commercial sources and distilled before use.

\section{Chromatography}

Flash chromatography was performed with Silicycle silica gel 60 (0.040-0.063 $\mu \mathrm{m}$ grade), phosphate buffered Silicycle silica gel 60 (0.040-0.063 $\mu \mathrm{m}$ grade) or basic alumina (Acros, Brockmann activity I, 50-200 $\mu \mathrm{m}, 60 \mathrm{~A}$ ). Analytical thin-layer chromatography was performed with commercial glass plates coated with $0.25 \mathrm{~mm}$ silica gel (E. Merck, Kieselgel 60 F254). Rf values reported were measured using a $5 \times 2 \mathrm{~cm}$ plate. Visualization was accomplished by UV light (254 $\mathrm{nm}$ ), or by dipping the plate in phosphomolybdic acid (PMA), aqueous potassium permanganate $\left(\mathrm{KMnO}_{4}\right)$ or ceric ammonium molybdate (CAM) solutions followed by heating.

\section{NMR Spectroscopy}

Proton nuclear magnetic resonance ( ${ }^{1} \mathrm{H}$ NMR), proton decoupled carbon-13 nuclear magnetic resonance $\left({ }^{13} \mathrm{C}\{1 \mathrm{H}\} \mathrm{NMR}\right)$ and proton decoupled fluorine-19 nuclear magnetic resonance $\left({ }^{19} \mathrm{~F}\right.$ $\{1 \mathrm{H}\}$ NMR) spectra were recorded on a Bruker AVANCEIII-400 spectrometer $\left(400 \mathrm{MHz},{ }^{1} \mathrm{H} ; 101\right.$ $\left.\mathrm{MHz},{ }^{13} \mathrm{C} ; 376 \mathrm{MHz},{ }^{19} \mathrm{~F}\right)$ or a Bruker AVANCEIII-600 spectrometer $\left(600 \mathrm{MHz},{ }^{1} \mathrm{H} ; 151 \mathrm{MHz},{ }^{13} \mathrm{C}\right)$. Chemical shifts are reported in parts per million (ppm). Spectra are referenced to residual chloroform $\left(\delta=7.26 \mathrm{ppm},{ }^{1} \mathrm{H} ; 77.16 \mathrm{ppm},{ }^{13} \mathrm{C}\right)$, residual benzene $\left(\delta=7.16 \mathrm{ppm},{ }^{1} \mathrm{H} ; 128.06 \mathrm{ppm}\right.$, $\left.{ }^{13} \mathrm{C}\right)$ or residual dimethyl sulfoxide $\left(\delta=2.50 \mathrm{ppm},{ }^{1} \mathrm{H} ; 39.52 \mathrm{ppm},{ }^{13} \mathrm{C}\right)$. Splitting patterns are designated as s, singlet; d, doublet; t, triplet; q, quartet; hept, heptuplet; m, multiplet, b, broad. Assignments were obtained by reference to COSY, HMQC, HMBC and NOESY correlations. All NMR data were recorded at $298 \mathrm{~K}$.

\section{Infrared Spectroscopy}

IR spectra were recorded on an Alpha-P Bruker FT-IR Spectrometer. Absorbance frequencies are reported in reciprocal centimeters $\left(\mathrm{cm}^{-1}\right)$ with indicated relative intensities: s (strong, 0-33\% T); $\mathrm{m}$ (medium, 34-66\% T); w (weak, 67-100\% T).

\section{Mass Spectrometry}


Electrospray-ionisation HRMS data were acquired on an Agilent LC-MS TOF (Multimode: ESI + APCI). High resolution mass data is given in $\mathrm{m} / \mathrm{z}$.

\section{Melting Points}

Melting points were measured on a Büchi B-540 and are uncorrected.

\section{X-Ray analyses}

X-ray analyses of compounds $\mathbf{2 b}, \mathbf{2 c}, \mathbf{5 k}$ and $\mathbf{7}$ were performed by Dr. R. Scopelliti and Dr. F. Fadaei Tirani at the EPF Lausanne.

\section{Elemental analyses}

Elemental analyses of compounds $\mathbf{2} \mathbf{g}, \mathbf{2} \mathbf{h}, \mathbf{3 b}$, and $\mathbf{5 l}$ were performed by Roxane Moinat at the elemental analysis platform of ISIC at the EPF Lausanne. 


\section{Rhodium and Iridium Precursors Synthesis}

1,5-Cyclooctadienerhodium $(\mathrm{I})$ chloride dimer $[\mathrm{Rh}(\mathrm{COD}) \mathrm{Cl}]_{2},{ }^{1}$ 1,5-Cyclooctadienerhodium $(\mathrm{I})$ hydroxide dimer $[\mathrm{Rh}(\mathrm{COD}) \mathrm{OH}]_{2}, \quad 2$ 1,5-Cyclooctadienerhodium(I) methoxide dimer $\mathrm{Rh}(\mathrm{COD}) \mathrm{OMe}]_{2}, \quad 2$ 1,5-Cyclooctadienerhodium(I) acetate dimer $[\mathrm{Rh}(\mathrm{COD}) \mathrm{OAc}]_{2}, \quad 3 \quad$ 1,5Cyclooctadienerhodium(I) benzoate dimer $[\mathrm{Rh}(\mathrm{COD}) \mathrm{OBz}]_{2},{ }^{4}$ Bicyclo[2.2.1] hepta-2,5-dienerhodium(I) chloride dimer $\left[\mathrm{Rh}(\mathrm{nbd}) \mathrm{Cl}_{2},{ }^{5}\right.$ Bicyclo[2.2.1] hepta-2,5-diene-rhodium(I) acetate dimer $[\mathrm{Rh}(\mathrm{nbd}) \mathrm{OAc}]_{2}, \quad 6 \quad 1,5$-Cyclooctadieneiridium(I) chloride dimer $[\operatorname{lr}(\mathrm{COD}) \mathrm{Cl}]_{2}, \quad 2 \quad 1,5-$ Cyclooctadieneiridium $(\mathrm{I})$ acetate dimer $[\operatorname{lr}(\mathrm{COD}) \mathrm{OAc}]_{2} .{ }^{6}$ were prepared following the literature procedure.

1,5-Cyclooctadienerhodium(I) pivalate dimer $[\mathrm{Rh}(\mathrm{COD}) \mathrm{OPiv}]_{2}$ was synthesized according to a procedure adapted from Chatt and Venanzi: ${ }^{7}$

To a flame-dried $20-\mathrm{mL}$ round bottom flask containing a stir bar and topped with a water-cooled reflux condenser under nitrogen was charged $\left[\mathrm{Rh}(\mathrm{COD}) \mathrm{Cl}_{2}(0.200 \mathrm{~g}, 0.406 \mathrm{mmol}, 1.0\right.$ equiv), potassium pivalate $(0.284 \mathrm{~g}, 2.03 \mathrm{mmol}, 5.0$ equiv) and degassed, dry acetone $(10 \mathrm{~mL}, 0.04 \mathrm{M})$. The suspension was stirred at reflux for $3 \mathrm{~h}$. The solution was cooled to $23^{\circ} \mathrm{C}$ and filtered through celite (eluted with $15 \mathrm{~mL}$ of DCM). The volatiles were removed under reduce pressure and the solid obtained was further dried on high vacuum overnight, affording $[\mathrm{Rh}(\mathrm{COD}) \mathrm{OPiv}]_{2}$ as a brown solid (0.249 g, 0.399 mmol, 98 \% yield). ${ }^{1} \mathrm{H}$ NMR: $\left(400 \mathrm{MHz}, \mathrm{C}_{6} \mathrm{D}_{6}\right) \delta 4.31-4.20(\mathrm{~m}, 2 \mathrm{H}), 4.16$ $4.08(\mathrm{~m}, 2 \mathrm{H}), 2.74-2.63(\mathrm{~m}, 2 \mathrm{H}), 2.33-2.21(\mathrm{~m}, 2 \mathrm{H}), 1.71-1.57(\mathrm{~m}, 2 \mathrm{H}), 1.55-1.42(\mathrm{~m}, 2 \mathrm{H})$. ${ }^{13} \mathrm{C}$ NMR: (101 MHz, $\left.\mathrm{C}_{6} \mathrm{D}_{6}\right) \delta$ 190.13, 79.90 (d, J = 14.7 Hz), 73.28 (d, J = 14.5 Hz), 39.80, 31.21, 30.34, 28.01. IR: (neat) 2953 (w), 2922 (w), 2880 (w), 2834 (w), 1563 (s), 1480 (w), 1455 (w), $1416(w), 1374(w), 1359(w), 1226(w), 954(w), 610(w)$. m.p.: $184^{\circ} \mathrm{C}$ (decomposition).

\section{Fulvene Synthesis}

The following fulvenes were prepared following literature procedures and the data characterization matched those previously reported.

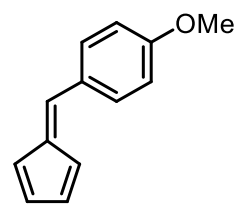

1-(cyclopenta-2,4-dien-1-ylidenemethyl)-4-methoxybenzene 1a. ${ }^{8}{ }^{1} \mathrm{H}$ NMR: (400 $\left.\mathrm{MHz}, \mathrm{CDCl}_{3}\right) \delta 7.58(\mathrm{~d}, \mathrm{~J}=8.7 \mathrm{~Hz}, 2 \mathrm{H}), 7.17(\mathrm{~d}, \mathrm{~J}=1.4 \mathrm{~Hz}, 1 \mathrm{H}), 6.94(\mathrm{~d}, \mathrm{~J}=8.8$ $\mathrm{Hz}, 2 \mathrm{H}), 6.73$ (ddd, J = 5.4, 2.1, $1.0 \mathrm{~Hz}, 1 \mathrm{H}), 6.67(\mathrm{dq}, \mathrm{J}=5.3,1.7 \mathrm{~Hz}, 1 \mathrm{H}), 6.49$ $(\mathrm{dt}, \mathrm{J}=5.1,1.7 \mathrm{~Hz}, 1 \mathrm{H}), 6.33(\mathrm{dt}, \mathrm{J}=5.1,1.8 \mathrm{~Hz}, 1 \mathrm{H}), 3.86(\mathrm{~s}, 3 \mathrm{H})$. 


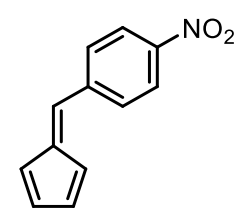

1-(cyclopenta-2,4-dien-1-ylidenemethyl)-4-methoxybenzene 1e. ${ }^{8}{ }^{1} \mathrm{H}$ NMR: (400 $\left.\mathrm{MHz}, \mathrm{C}_{6} \mathrm{D}_{6}\right) \delta 7.71(\mathrm{~d}, J=8.8 \mathrm{~Hz}, 2 \mathrm{H}), 6.88(\mathrm{~d}, J=8.8 \mathrm{~Hz}, 2 \mathrm{H}), 6.55-6.47(\mathrm{~m}$, 2H), 6.40 (dt, $J=5.3,1.6 \mathrm{~Hz}, 1 \mathrm{H}), 6.34(\mathrm{dt}, J=5.4,1.6 \mathrm{~Hz}, 1 \mathrm{H}), 6.13$ (dt, $J=5.3$, $1.8 \mathrm{~Hz}, 1 \mathrm{H})$.

$\overbrace{}^{\mathrm{n} B \mathrm{Bu}}$

5-pentylidenecyclopenta-1,3-diene 1f. ${ }^{9}{ }^{1} \mathrm{H}$ NMR: $\left(400 \mathrm{MHz}, \mathrm{C}_{6} \mathrm{D}_{6}\right) \delta 6.54$ (t, J = $1.7 \mathrm{~Hz}$, $2 \mathrm{H}), 6.47(\mathrm{dd}, \mathrm{J}=5.1,1.8 \mathrm{~Hz}, 1 \mathrm{H}), 6.24(\mathrm{dt}, \mathrm{J}=5.2,1.7 \mathrm{~Hz}, 1 \mathrm{H}), 6.09(\mathrm{t}, \mathrm{J}=7.9 \mathrm{~Hz}, 1 \mathrm{H})$, $2.24(\mathrm{q}, \mathrm{J}=7.4 \mathrm{~Hz}, 2 \mathrm{H}), 1.25-1.07(\mathrm{~m}, 4 \mathrm{H}), 0.77(\mathrm{t}, \mathrm{J}=7.2 \mathrm{~Hz}, 3 \mathrm{H})$.

$\int^{\mathrm{Cy}}$ (cyclopenta-2,4-dien-1-ylidenemethyl)cyclohexane 1g. ${ }^{9}{ }^{1} \mathrm{H} \mathrm{NMR}:\left(400 \mathrm{MHz}, \mathrm{C}_{6} \mathrm{D}_{6} \delta 6.55\right.$ (d, J = 1.7 Hz, 2H), $6.52-6.43(\mathrm{~m}, 1 \mathrm{H}), 6.25(\mathrm{~d}, \mathrm{~J}=5.1 \mathrm{~Hz}, 1 \mathrm{H}), 6.03(\mathrm{~d}, \mathrm{~J}=9.9 \mathrm{~Hz}, 1 \mathrm{H})$, $2.56(\mathrm{qt}, \mathrm{J}=10.5,3.6 \mathrm{~Hz}, 1 \mathrm{H}), 1.72-1.45(\mathrm{~m}, 5 \mathrm{H}), 1.28-0.88(\mathrm{~m}, 5 \mathrm{H})$.

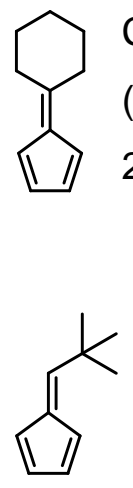
Cyclopenta-2,4-dien-1-ylidenecyclohexane 1h. ${ }^{10}{ }^{1} \mathrm{H} \mathrm{NMR}$ : $\left(400 \mathrm{MHz}, \mathrm{C}_{6} \mathrm{D}_{6}\right) \delta 6.66-6.61$ $(\mathrm{m}, 2 \mathrm{H}), 6.61-6.56(\mathrm{~m}, 2 \mathrm{H}), 2.39-2.27(\mathrm{~m}, 4 \mathrm{H}), 1.46-1.36(\mathrm{~m}, 4 \mathrm{H}), 1.34-1.22(\mathrm{~m}$, $2 \mathrm{H})$.

5-(2,2-dimethylpropylidene)cyclopenta-1,3-diene 1i. ${ }^{10}{ }^{1} \mathrm{H} \mathrm{NMR}$ : $\left(400 \mathrm{MHz}, \mathrm{CDCl}_{3}\right) \delta$ 6.68 (ddt, $J=5.4,2.0,0.9 \mathrm{~Hz}, 1 \mathrm{H}), 6.59(\mathrm{dq}, J=5.4,1.7 \mathrm{~Hz}, 1 \mathrm{H}), 6.47-6.43(\mathrm{~m}, 1 \mathrm{H})$, $6.40(\mathrm{dt}, J=5.3,1.5 \mathrm{~Hz}, 1 \mathrm{H}), 6.16(\mathrm{dt}, J=5.2,1.8 \mathrm{~Hz}, 1 \mathrm{H}), 1.29(\mathrm{~s}, 9 \mathrm{H})$.

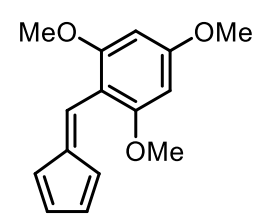

2-(cyclopenta-2,4-dien-1-ylidenemethyl)-1,3,5-trimethoxybenzene 1j. ${ }^{11}{ }^{1} \mathrm{H}$ NMR: $\left(400 \mathrm{MHz}, \mathrm{C}_{6} \mathrm{D}_{6}\right) \delta 7.53-7.41(\mathrm{~m}, 1 \mathrm{H}), 6.81(\mathrm{dd}, \mathrm{J}=5.4,1.9 \mathrm{~Hz}, 1 \mathrm{H}), 6.66-6.57$ $(\mathrm{m}, 1 \mathrm{H}), 6.50-6.44(\mathrm{~m}, 2 \mathrm{H}), 6.01(\mathrm{~s}, 2 \mathrm{H}), 3.33(\mathrm{~s}, 3 \mathrm{H}), 3.23(\mathrm{~s}, 6 \mathrm{H})$.

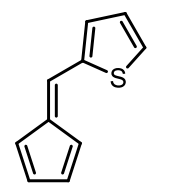

2-(cyclopenta-2,4-dien-1-ylidenemethyl)thiophene 1k. ${ }^{12}{ }^{1} \mathrm{H} \mathrm{NMR}:\left(400 \mathrm{MHz}, \mathrm{C}_{6} \mathrm{D}_{6}\right) \delta$ 7.05 (ddt, $J=5.4,2.0,0.9 \mathrm{~Hz}, 1 \mathrm{H}), 6.85(\mathrm{~d}, J=1.4 \mathrm{~Hz}, 1 \mathrm{H}), 6.82-6.76(\mathrm{~m}, 2 \mathrm{H}), 6.56$ (ddt, $J=6.5,5.0,2.7 \mathrm{~Hz}, 2 \mathrm{H}$ ), 6.41 (dt, $J=5.2,1.7 \mathrm{~Hz}, 1 \mathrm{H}$ ), 6.21 (dt, $J=5.1,1.8 \mathrm{~Hz}$, $1 \mathrm{H})$.

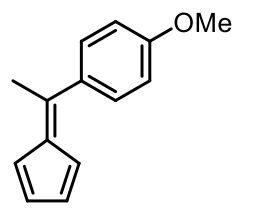

1-(1-(cyclopenta-2,4-dien-1-ylidene)ethyl)-4-methoxybenzene 8. ${ }^{9}{ }^{1} \mathrm{H}$ NMR: (400 $\left.\mathrm{MHz}, \mathrm{CDCl}_{3}\right) \delta 7.37(\mathrm{~d}, \mathrm{~J}=8.8 \mathrm{~Hz}, 2 \mathrm{H}), 6.93(\mathrm{~d}, \mathrm{~J}=8.8 \mathrm{~Hz}, 2 \mathrm{H}$ ), 6.64 (ddd, J = 
5.3, 2.1, $1.4 \mathrm{~Hz}, 1 \mathrm{H}), 6.57(\mathrm{dt}, \mathrm{J}=5.2,1.8 \mathrm{~Hz}, 1 \mathrm{H}), 6.50(\mathrm{dt}, \mathrm{J}=5.4,1.8 \mathrm{~Hz}, 1 \mathrm{H}), 6.25$ (ddd, J = 5.3, 2.1, $1.5 \mathrm{~Hz}, 1 \mathrm{H}), 3.86(\mathrm{~s}, 3 \mathrm{H}), 2.54(\mathrm{~s}, 3 \mathrm{H})$. 


\section{Crystallographic Data}

Crystallographic data for the structures reported in this paper have been deposited at the Cambridge Crystallographic Data Center (CCDC) as Supplementary Publication No. CCDC2022942 (2b), CCDC2022943 (2c), and CCDC2022944 (5k) and CCDC2022940 (7). Copies of the data can be obtained free of charge on application to the CCDC via https://www.ccdc.cam.ac.uk/data_request/cif.

\section{Crystallization methods:}

- X-Ray quality crystals of $\mathbf{2} \mathbf{b}$ were obtained by slow vapor diffusion of methanol in a solution of the complex $(5 \mathrm{mg})$ in chloroform $(0.1 \mathrm{ml})$ at room temperature for two days.

- X-Ray quality crystals of $\mathbf{2 c}$ were obtained by slow vapor diffusion of ethanol in a solution of the complex $(5 \mathrm{mg})$ in chloroform $(0.1 \mathrm{ml})$ at room temperature for several days

- X-Ray quality crystals of $\mathbf{5 k}$ were obtained by slow vapor diffusion of toluene in a solution of the complex $(5 \mathrm{mg})$ in chloroform $(0.1 \mathrm{ml})$ at room temperature for several days

- X-Ray quality crystals of $\mathbf{7}$ were obtained by slow vapor diffusion of hexane in a solution of the complex $(5 \mathrm{mg})$ in dichloromethane $(0.1 \mathrm{ml})$ at room temperature for several days. 


\section{References}

(1) Ashfeld, A.; Judd, A. Encyclopedia of Reagents for Organic Synthesis, DOI: 10.1002/9780470842898.rn00699.

(2) Kirschner, S. Inorg. Synth., Wiley-VCH Verlag GmbH \& Co. KGaA, New-York, 1985, pp126.

(3) Filloux, C.; Rovis, T. Rh(I)-Biphosphine Catalyzed Asymmetric, Intramolecular Hydroheteroarylation of a-Substituted Acrylate Derivatives. J. Am. Chem. Soc., 2015, 137, 508-517.

(4) Crabtree, R.; Gautier, A.; Giordano, G.; Khan, T. The Preparation and Some Catalytic Properties of a Number of Rhodium(I) Diolefin Complexes. J. Organomet. Chem. 1977, 141, 113-121.

(5) Baghurst, D.; Mingos, M.; Watson, M. Application of Microwave Dielectric Loss Heating Effects for the Rapid and Convenient Synthesis of Organometallic Compounds. J. Organomet. Chem. 1989, 368, 43-45.

(6) Haszeldine, R.; Lunt, R.; Parish, R. Organic Reactions Involving Transition Metals. Part IV. Carboxylatodiene Complexes of Rhodium(I) and Iridium(I). J. Chem. Soc. A, 1971, 3696-3698.

(7) Chatt, J.; Venanzi, L. Olefin Co-Ordination Compounds. Part VI. Diene Complex of Rhodium(I). J. Chem. Soc. 1957, 4735-4741.

(8) Coşkun, N.; Erden, I. An Efficient Catalytic Method for Fulvene Synthesis. Tetrahedron, 2011, 67, 8607-8614.

(9) Sieverding, P.; Osterbrink, J.; Besson, C.; Kögerler, P. Kinetics and Mechanism of Pyrrolidine Buffer-Catalyzed Fulvene Formation. J. Org. Chem. 2019, 84, 486-494.

(10) Stone, K.; Little, D. An Exceptionally Simple and Efficient Method for the Preparation of a Wide Varietz of Fulvenes. J. Org. Chem, 1984, 49, 1849-1853.

(11) Sirignano, A.; Saturnino, C.; Botta, A.; Sinicropi, M.; Lappano, R.; Caruso, A.; Pisano, A.; D.; Lappano, R.; Maggiolini, M.; Longo, P.Synthesis, Characterization and Cytotoxic Activity on Breast Cancer Cells of New Half-Titanocene. Bioorg. Med. Chem. Lett. 2013 23, 3458-3462.

(12) Cini, M.; Bradshaw, T.; Woodward, S.; Lewis, W. Asymmetric Pentafulvene Carbometalation-Access to Enantiopure Titanocene Dichlorides of Biological Relevance. Angew. Chem. Int. Ed. 2015, 54, 14182-14182. 


\section{NMR spectra}
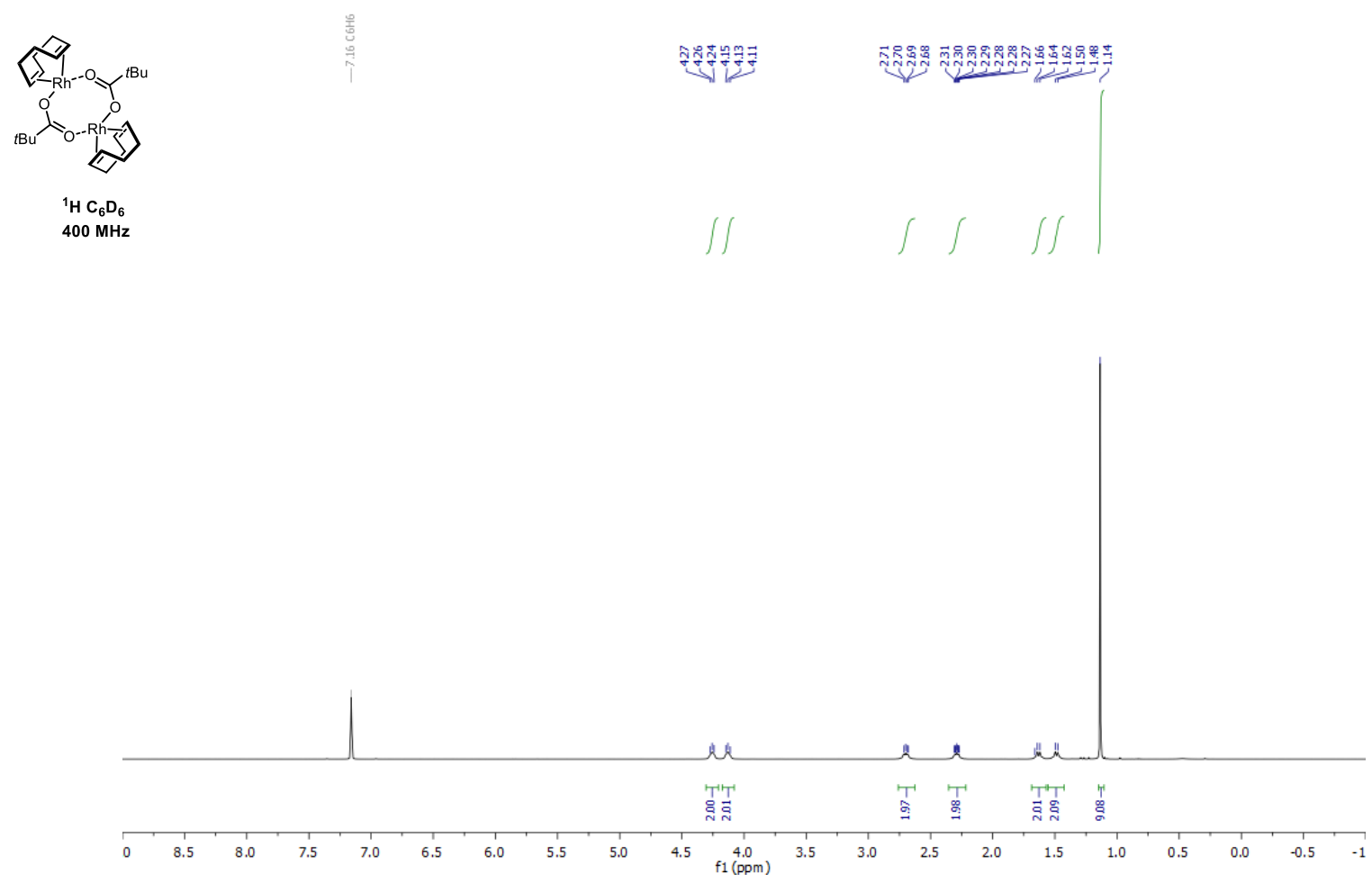

Figure S1: [Rh(COD)OPiv]2, ${ }^{1} \mathrm{H}$ NMR

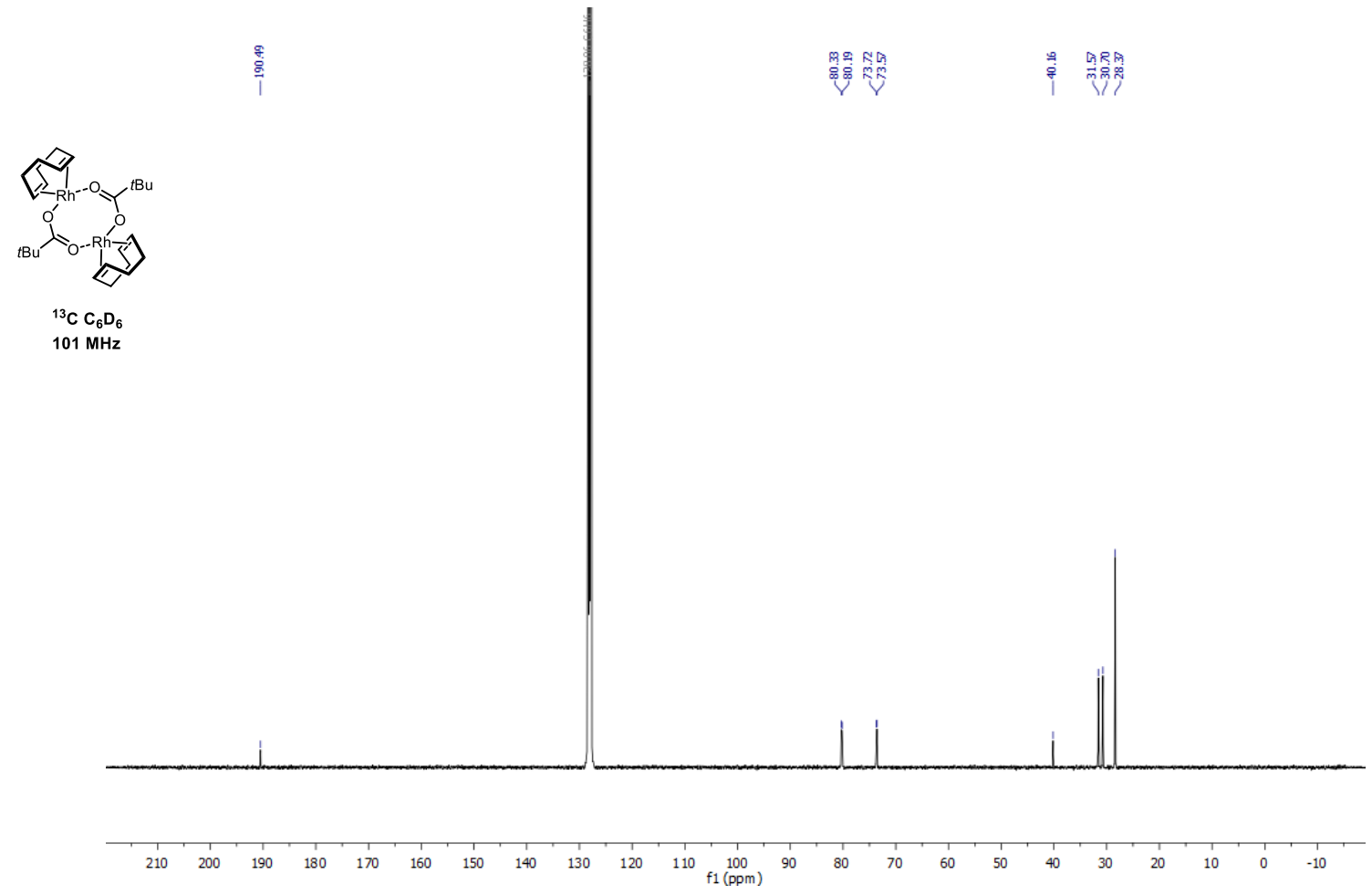

Figure S2: $[R h(C O D) O P i v] 2,{ }^{13} \mathrm{C} N M R$ 

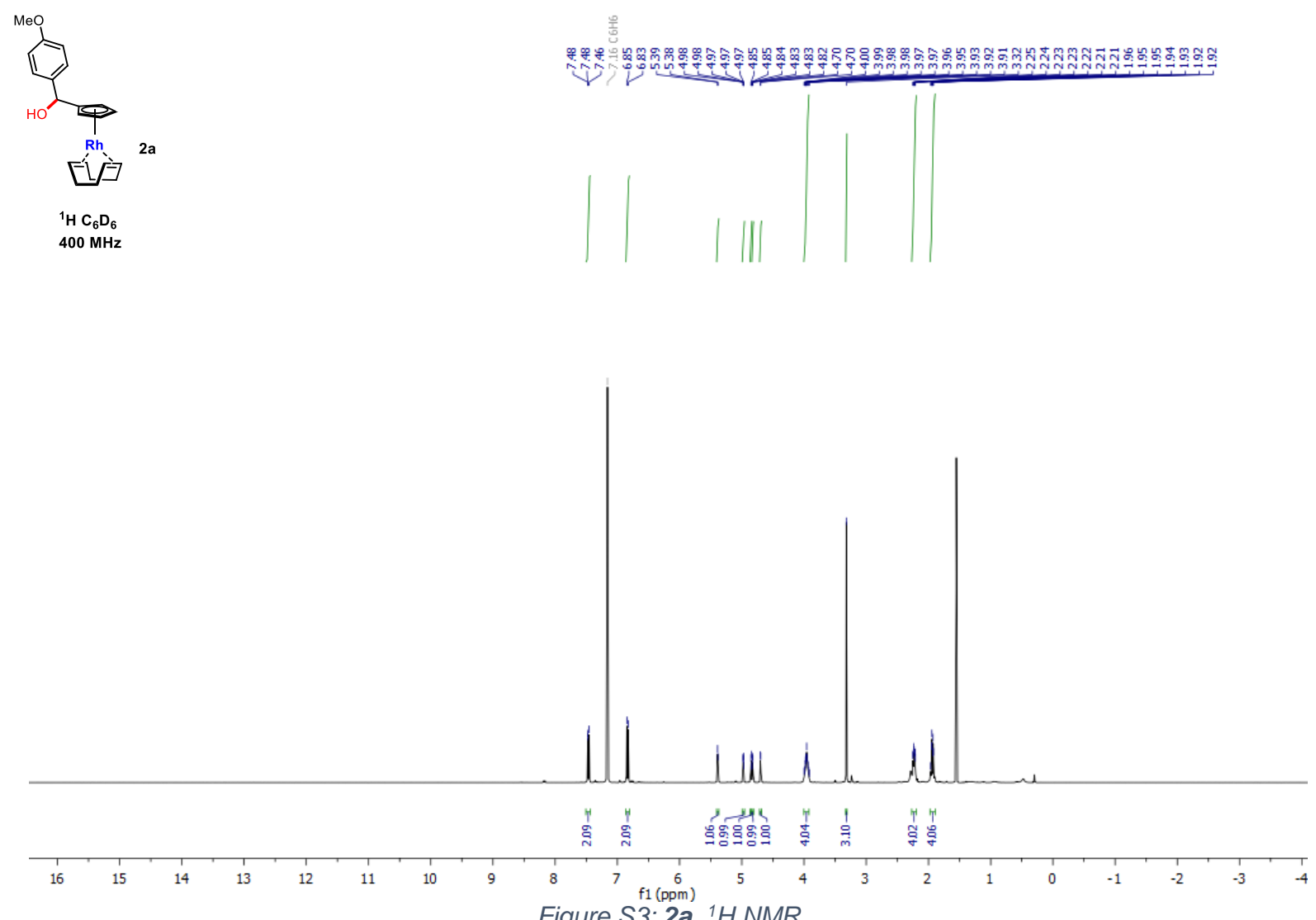

Figure S3: 2a, ${ }^{1}$ H NMR

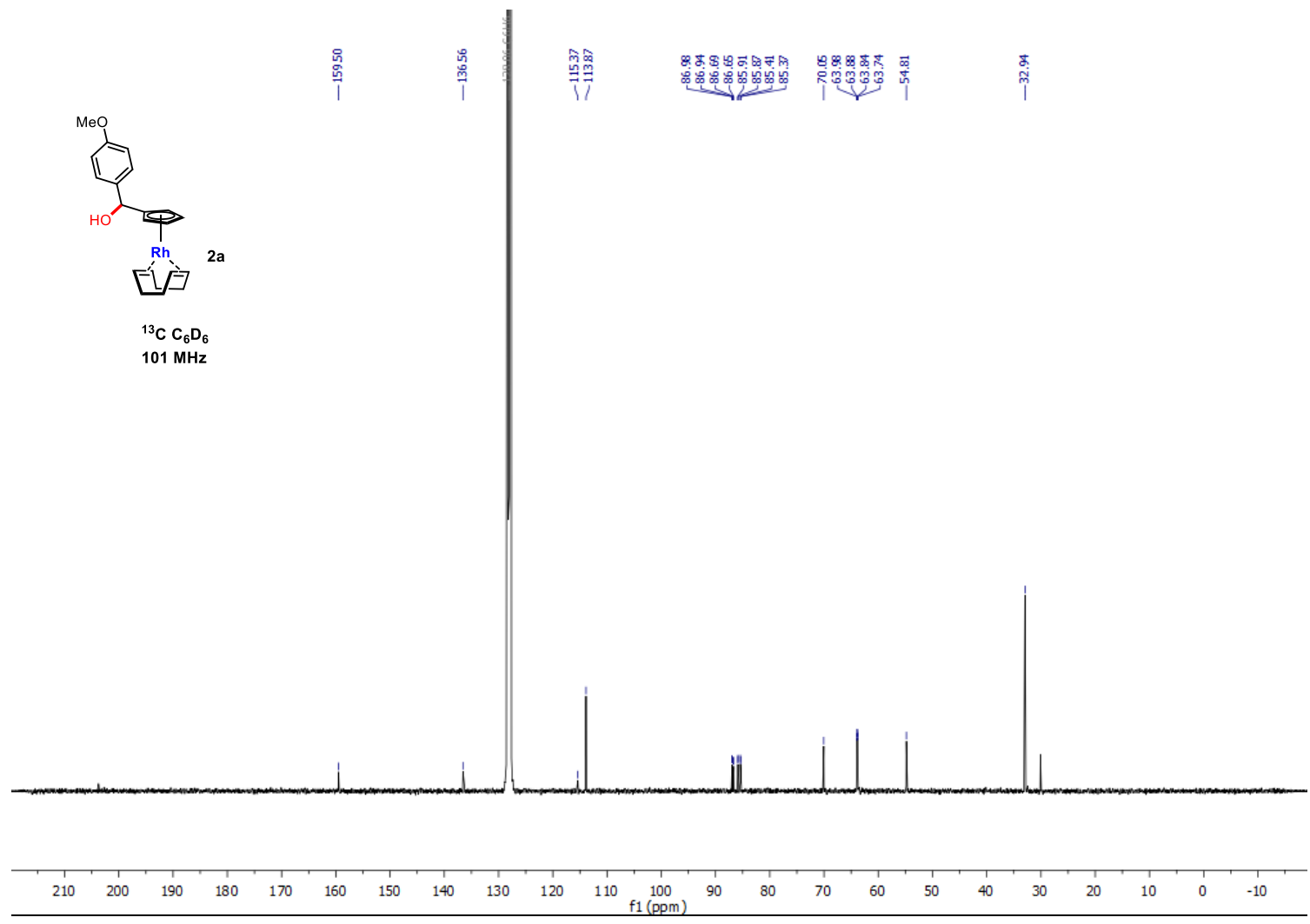

Figure S4: $2 \mathbf{a},{ }^{13} \mathrm{C} N M R$ 

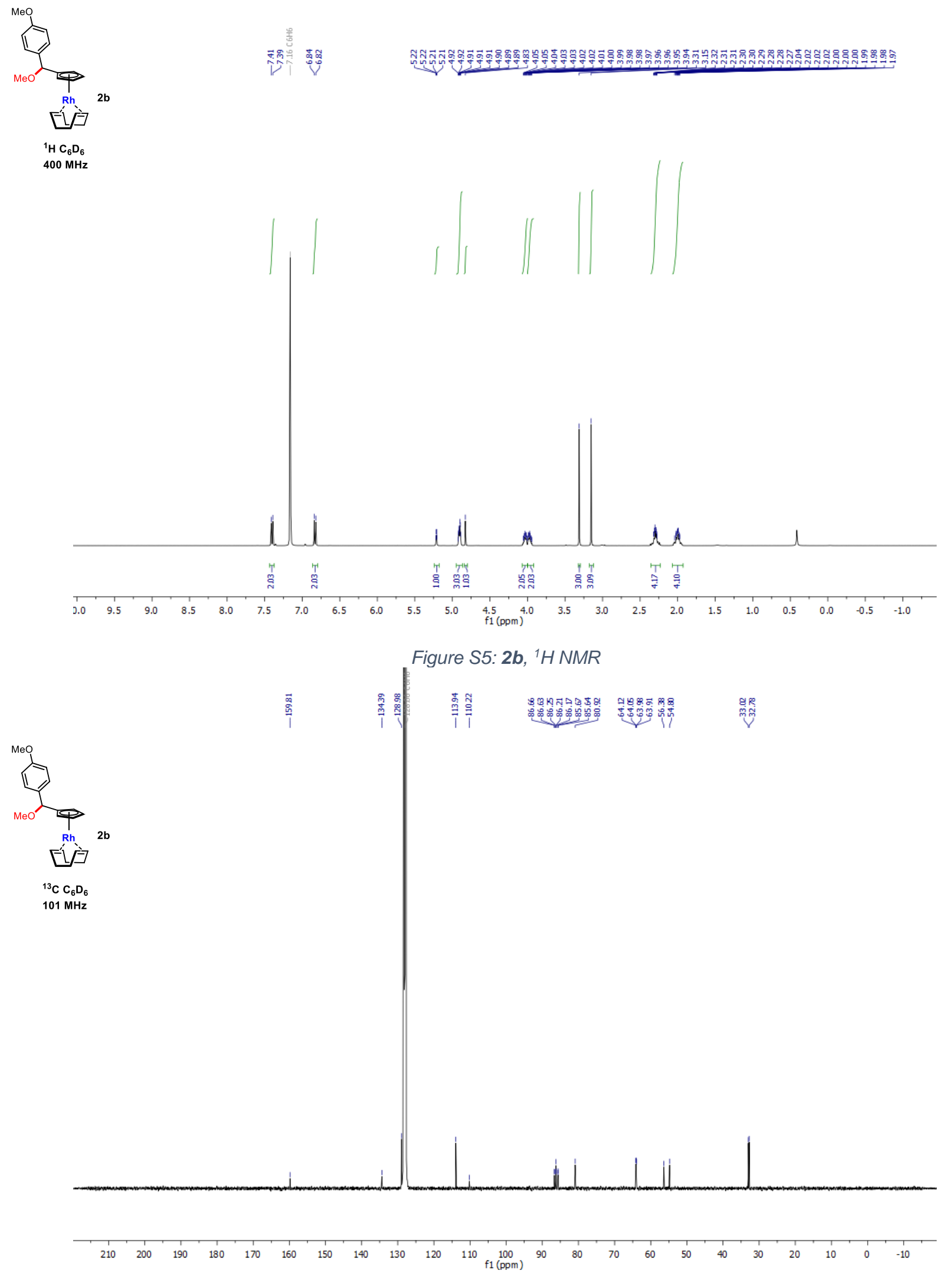

Figure S6: $2 b,{ }^{13} \mathrm{C} N M R$ 

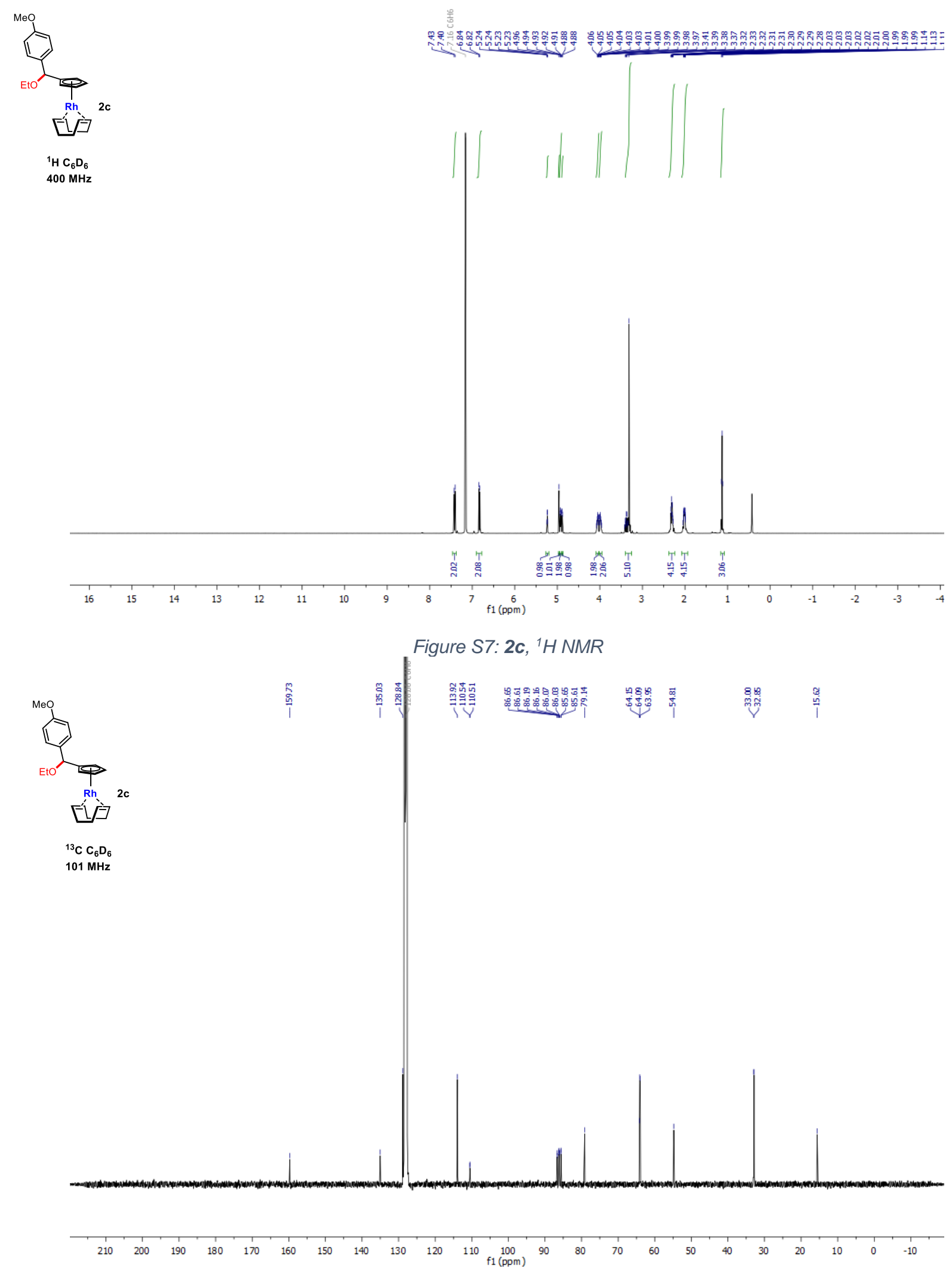

Figure S8: $2 c,{ }^{13} \mathrm{C}$ NMR 

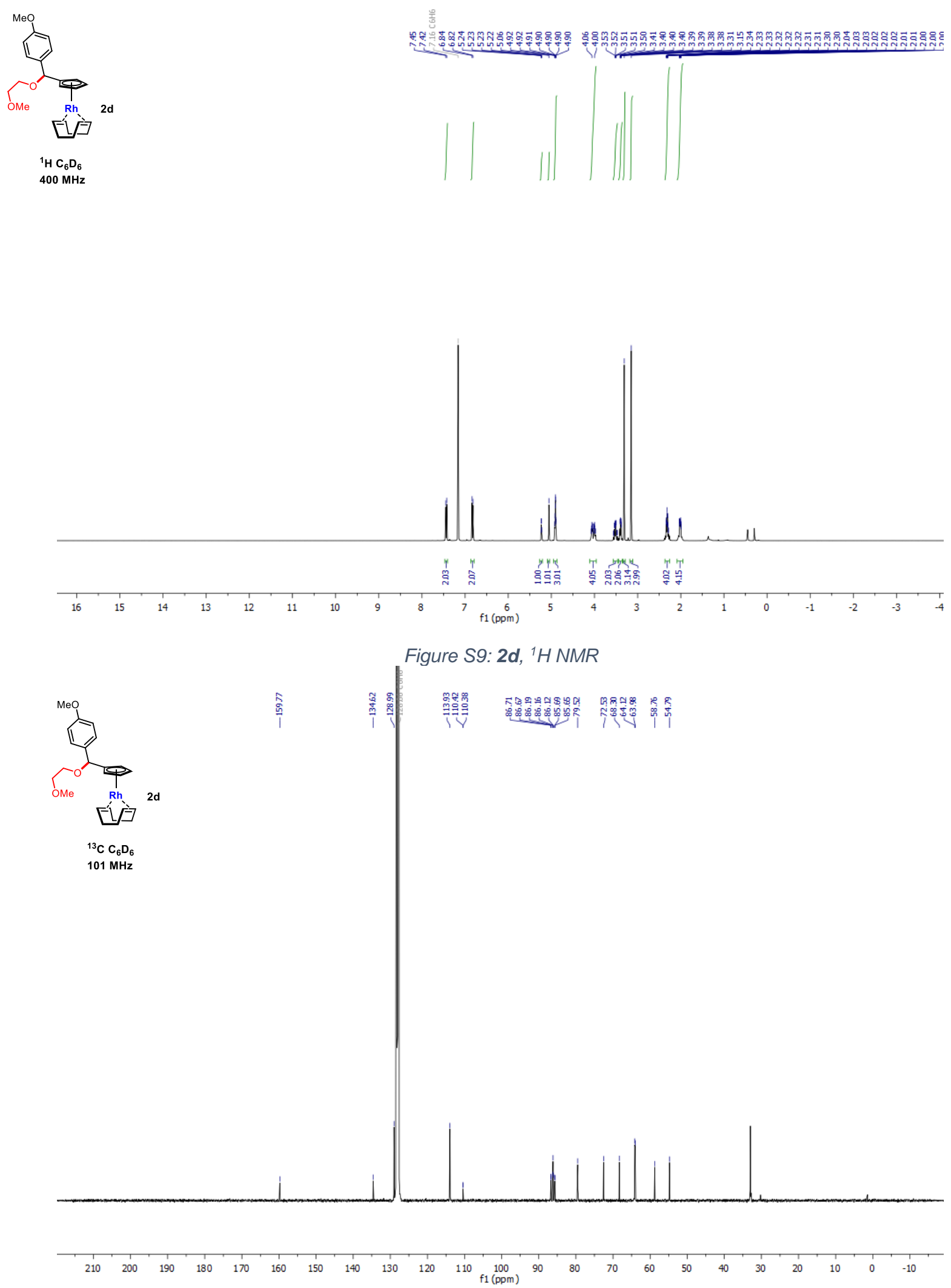

Figure S10: $2 d,{ }^{13} \mathrm{C}$ NMR 

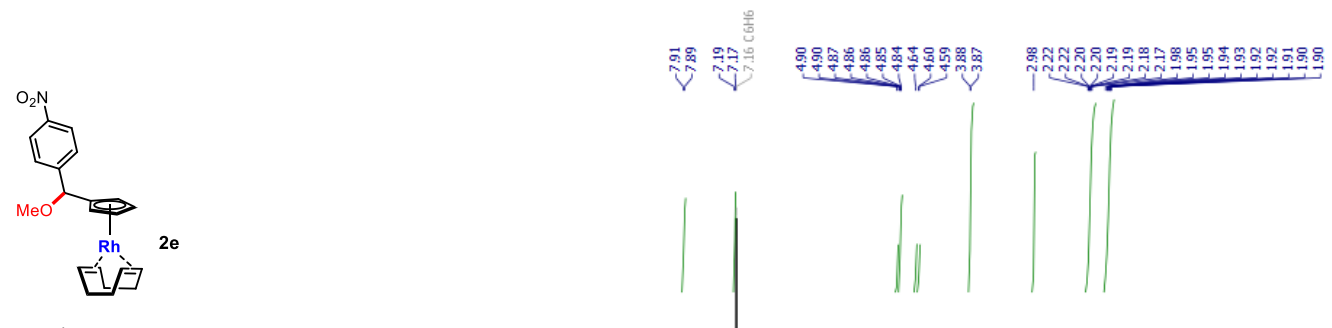

${ }^{1} \mathrm{H} \mathrm{C}_{6} \mathrm{D}_{6}$

$400 \mathrm{MHz}$
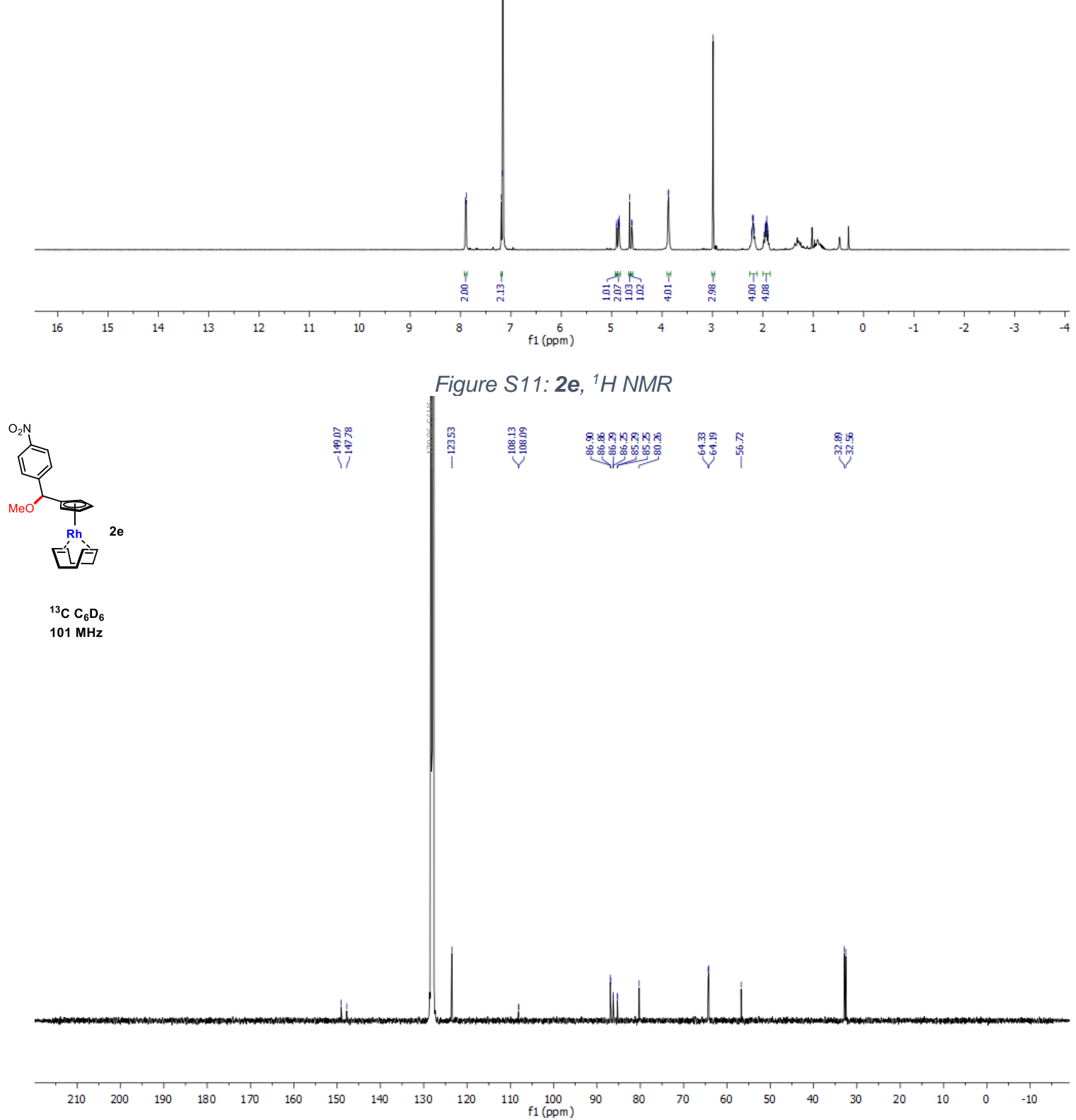

Figure S12: $2 e,{ }^{13} \mathrm{C}$ NMR 

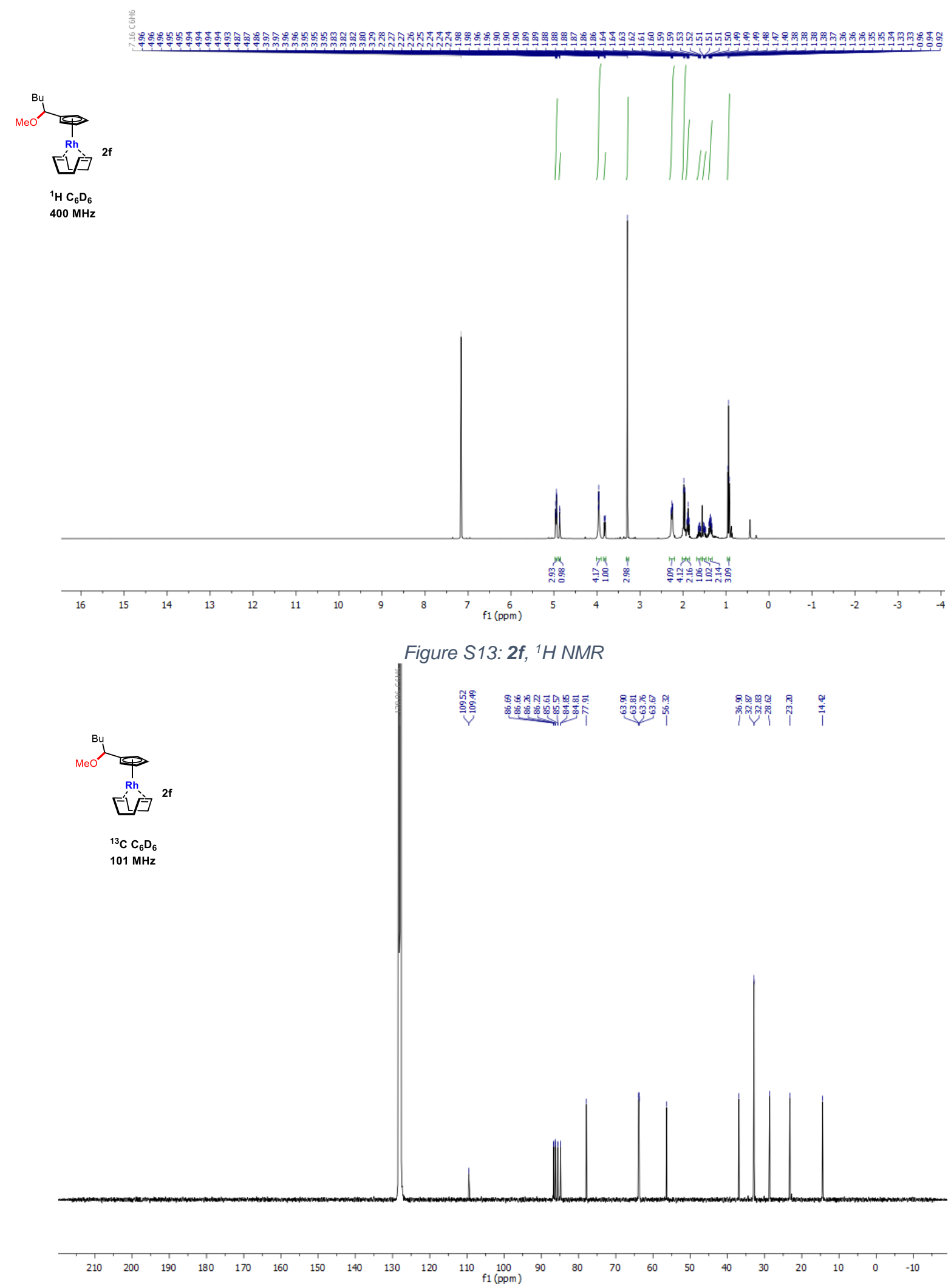

Figure S14: 2f, ${ }^{13} \mathrm{C}$ NMR 
赛

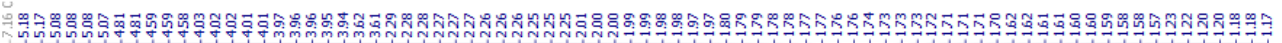
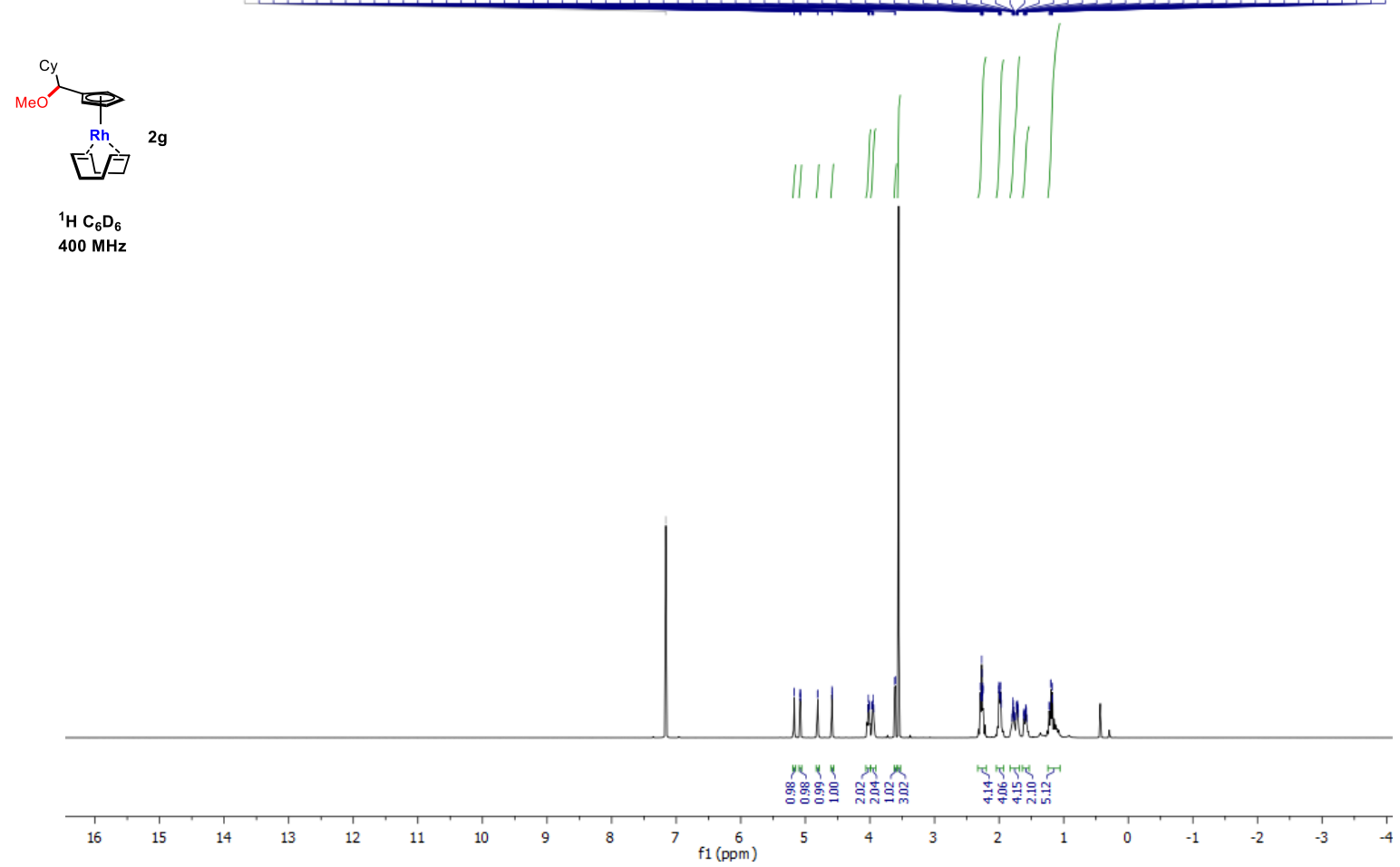

Figure S15: 2g, ${ }^{1} H$ NMR
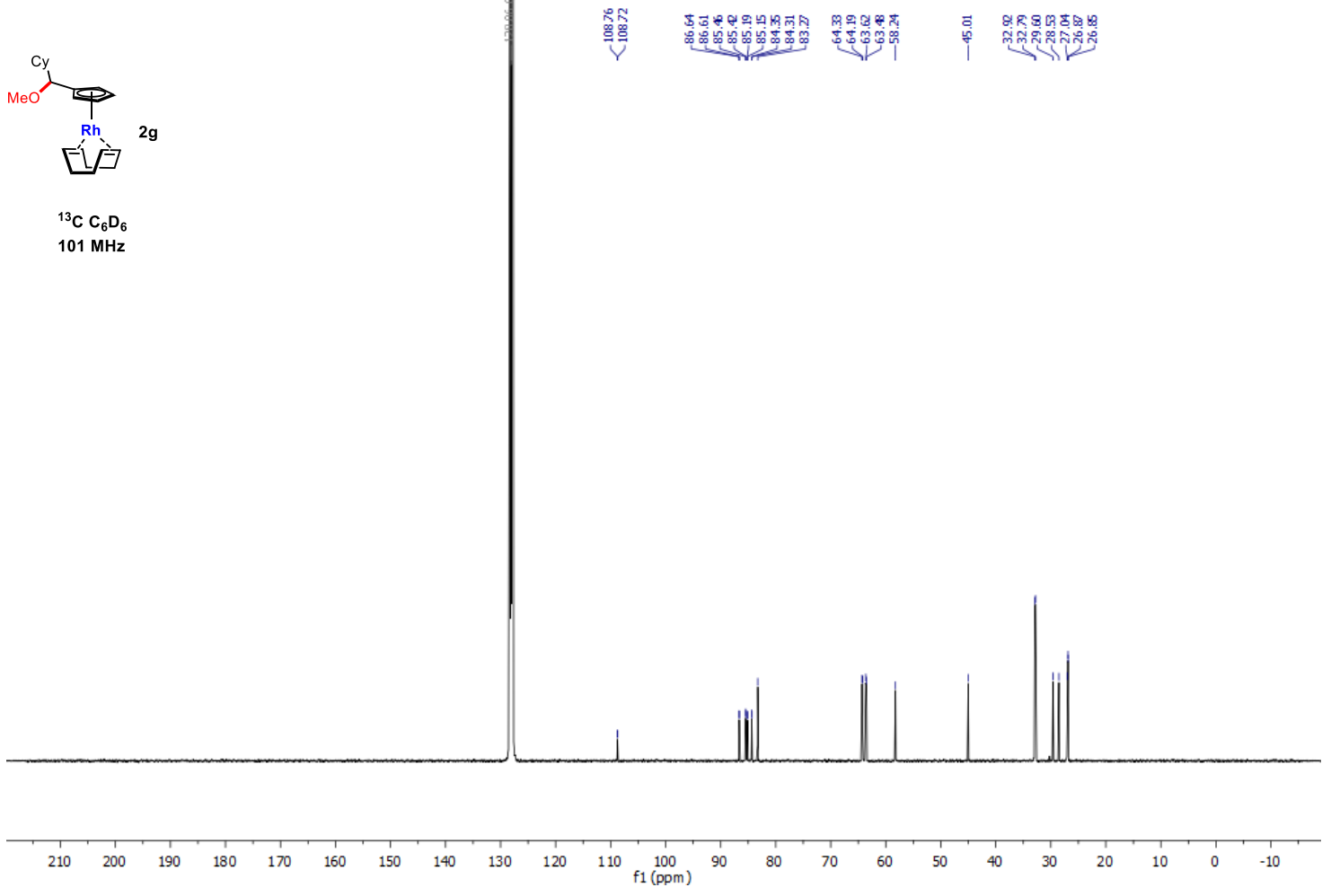

Figure S16: 2g, ${ }^{13} \mathrm{C}$ NMR

S16 

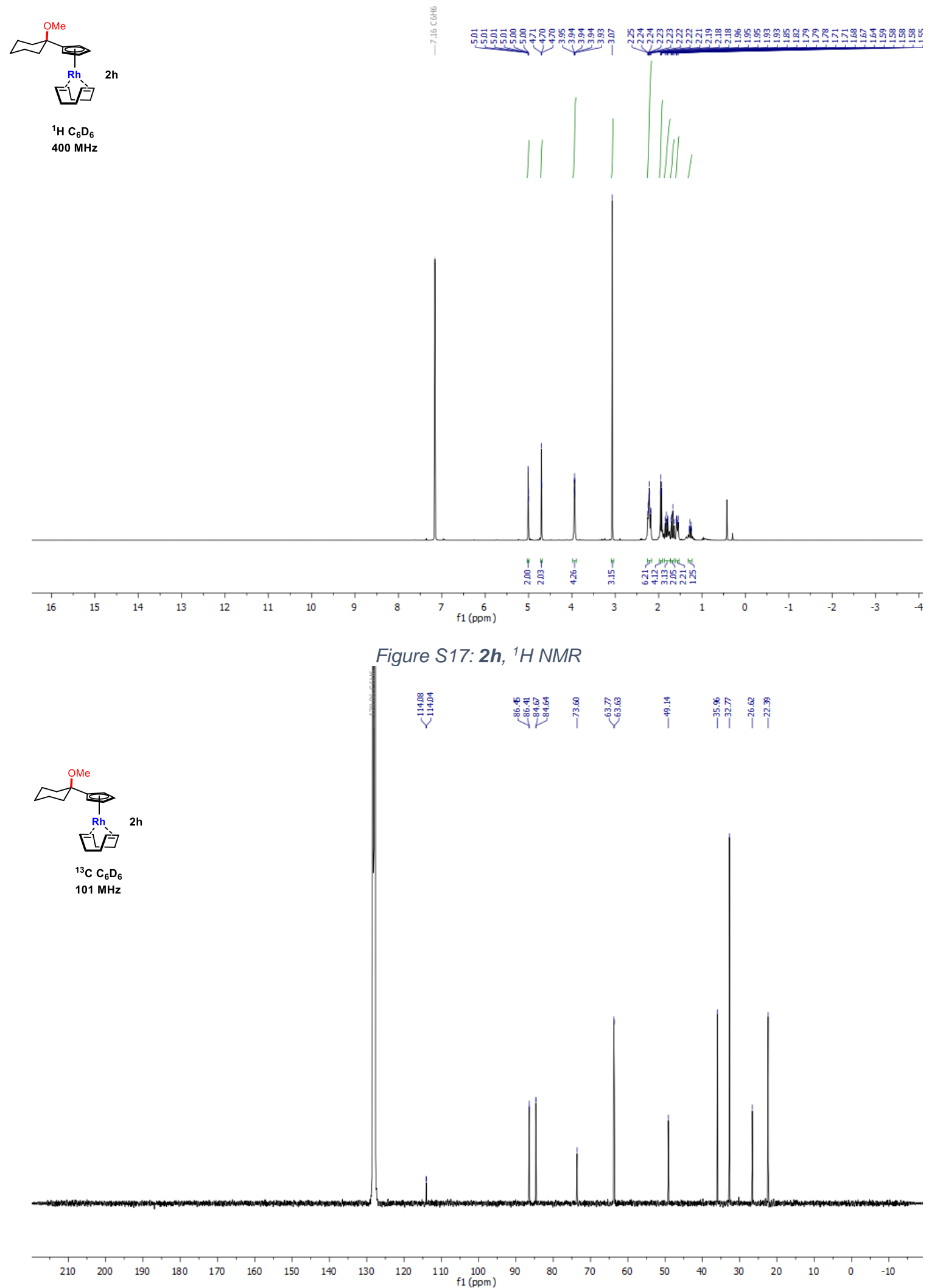

Figure S18: $2 h,{ }^{13} \mathrm{C}$ NMR 


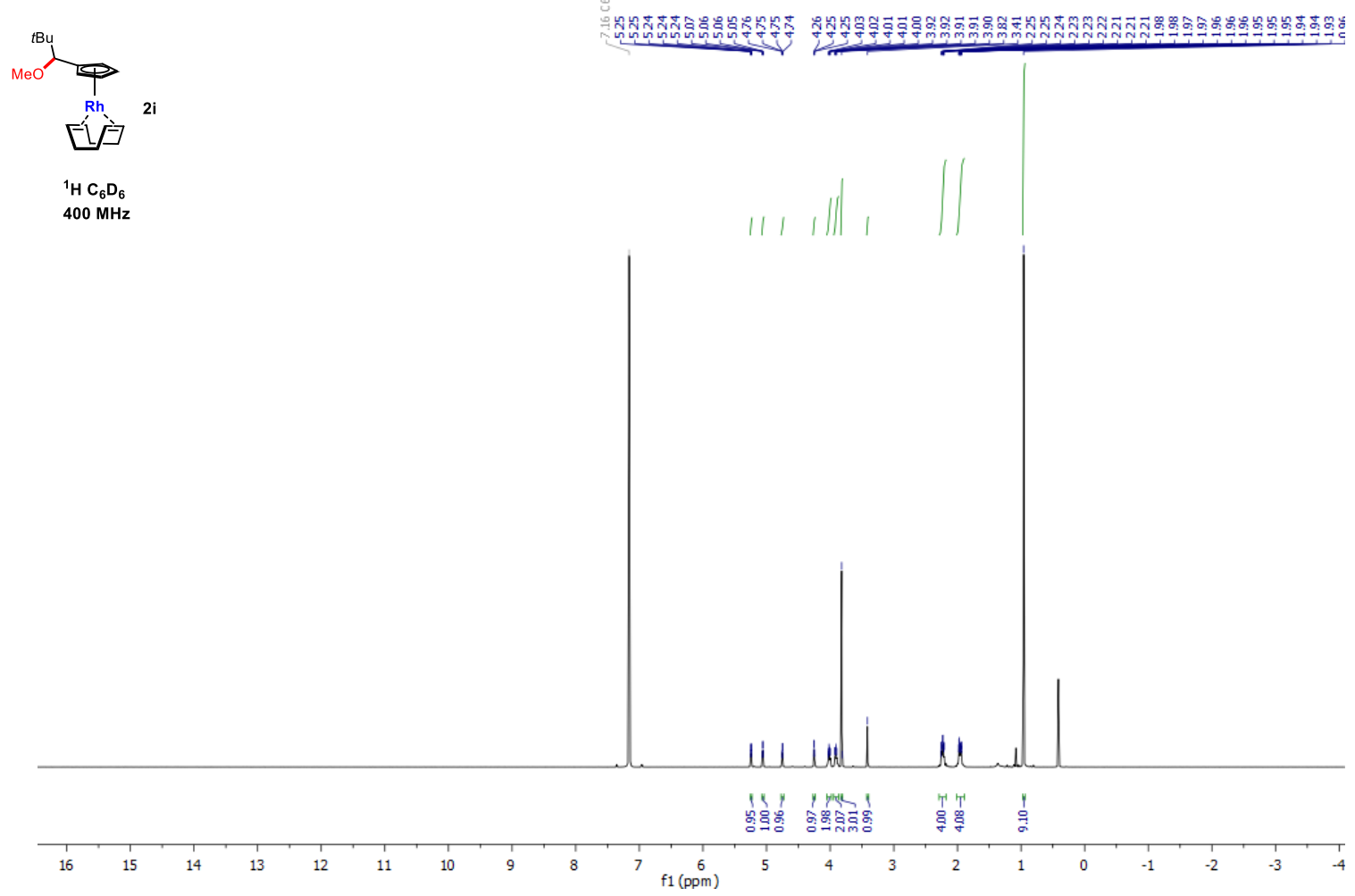

Figure S19: $2 i,{ }^{1} H$ NMR

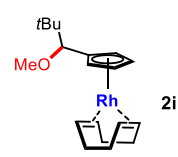

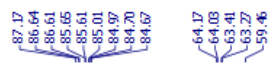

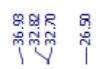

${ }^{13} \mathrm{C} \mathrm{C}_{6} \mathrm{D}_{6}$
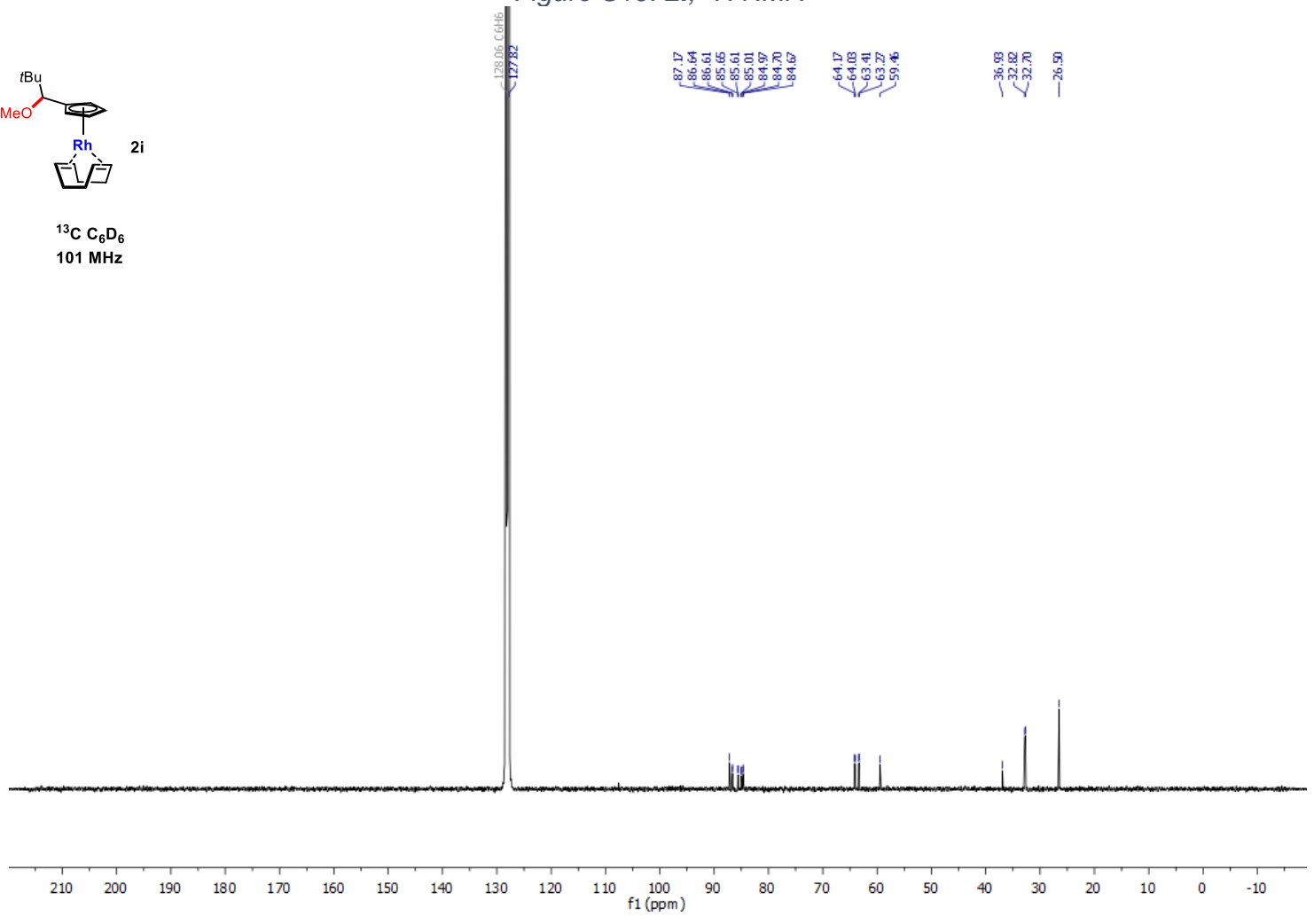

Figure S20: $2 i,{ }^{13} \mathrm{C}$ NMR

S18 

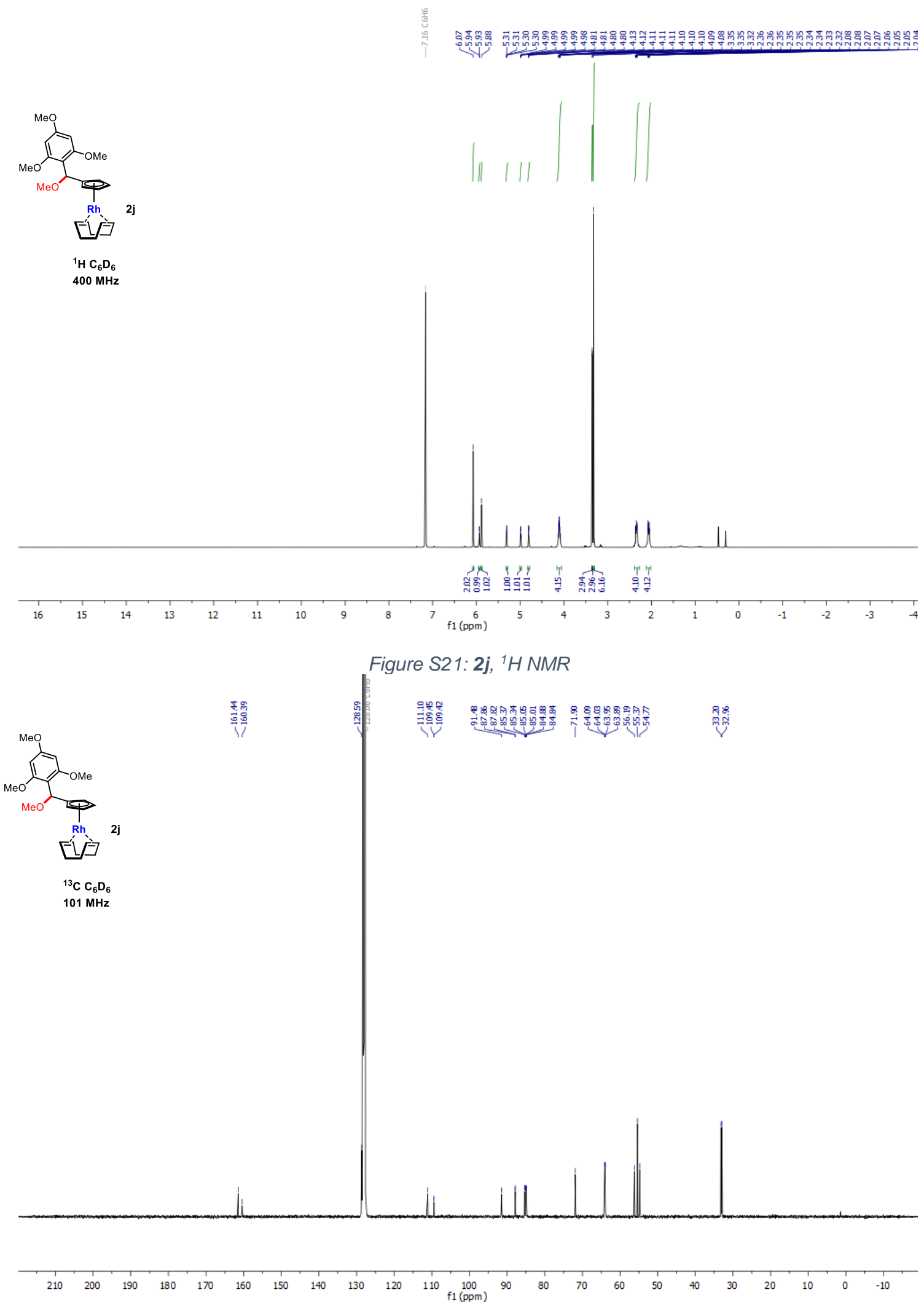

Figure S22: $2 \mathrm{j},{ }^{13} \mathrm{C}$ NMR 

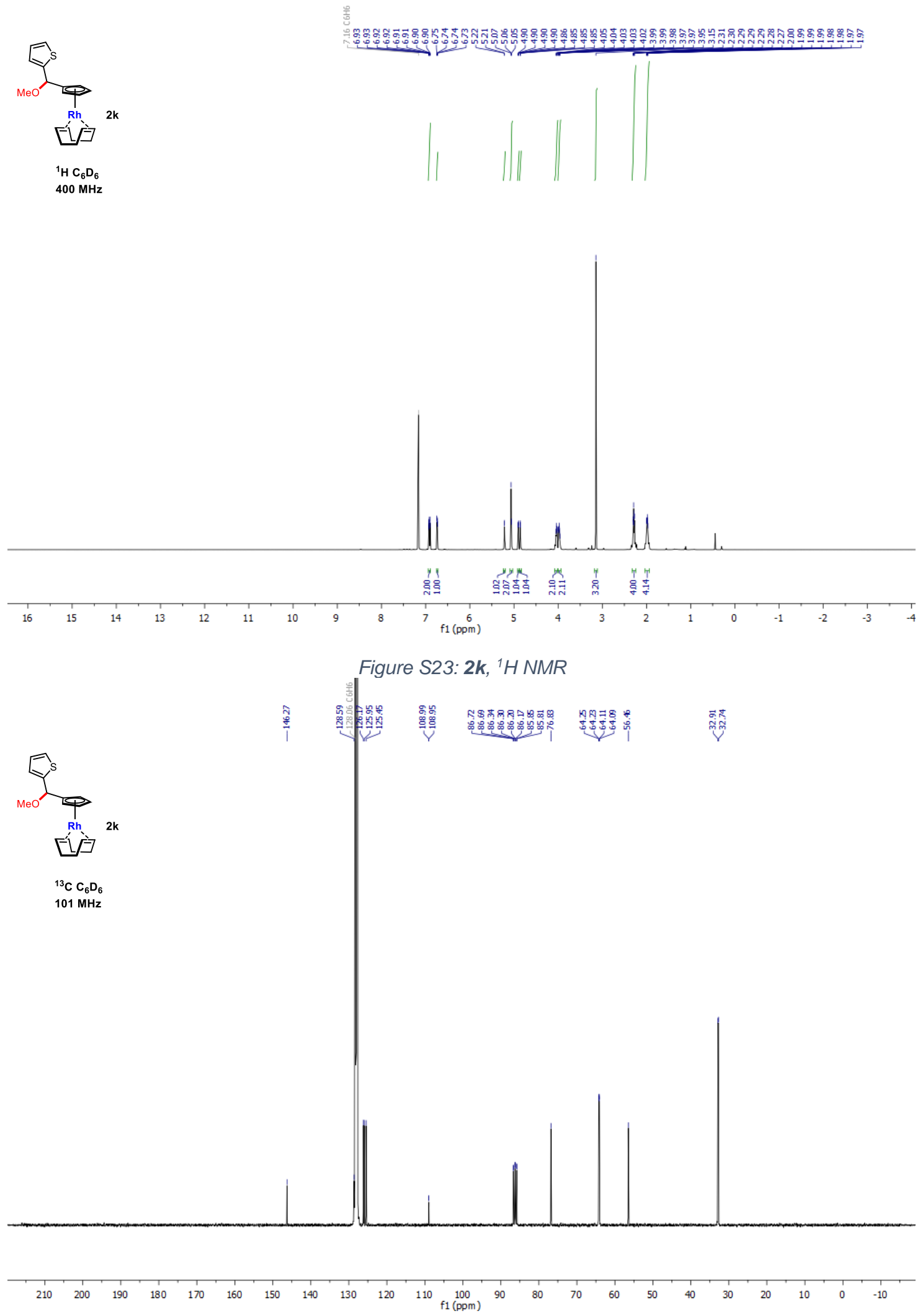

Figure S24: $2 \boldsymbol{k},{ }^{13} \mathrm{C}$ NMR 


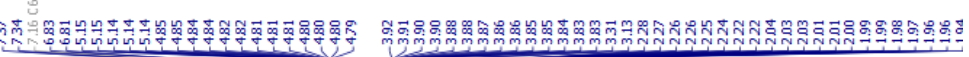
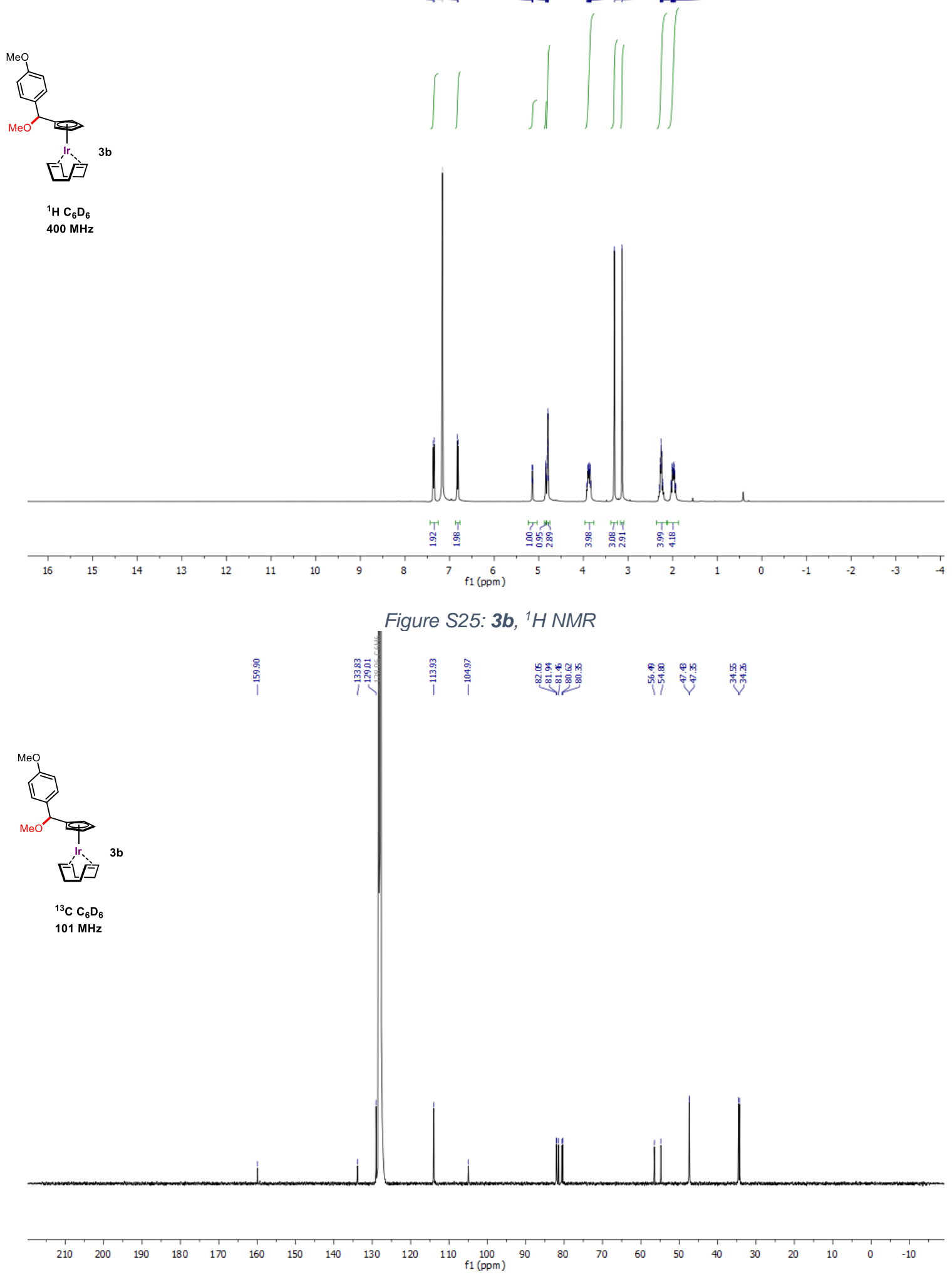

Figure S26: $3 b,{ }^{13} \mathrm{C} N M R$

S21 

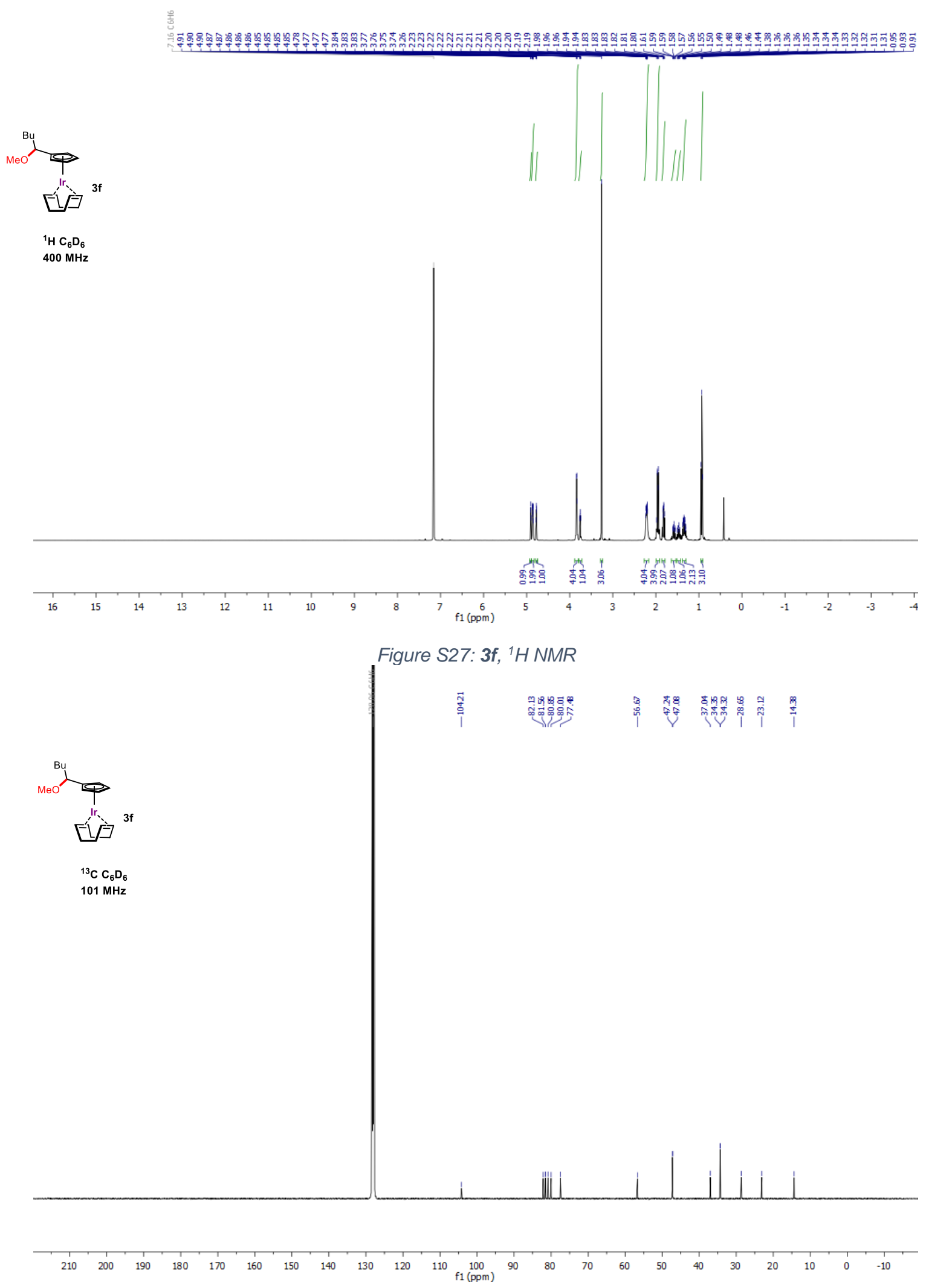

Figure S28: $3 f,{ }^{13} \mathrm{C}$ NMR 

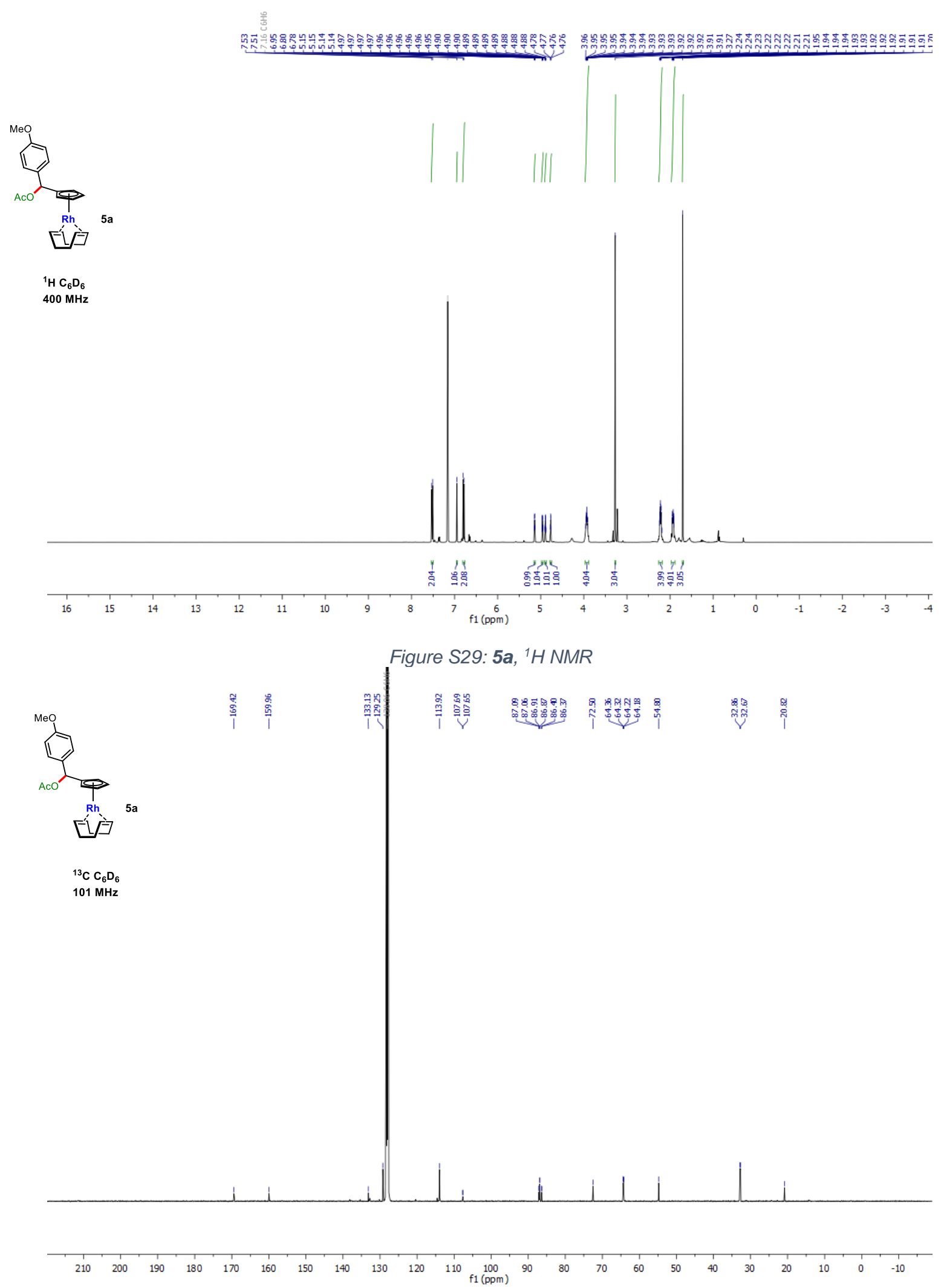

Figure S30: 5a, ${ }^{13} \mathrm{C}$ NMR 

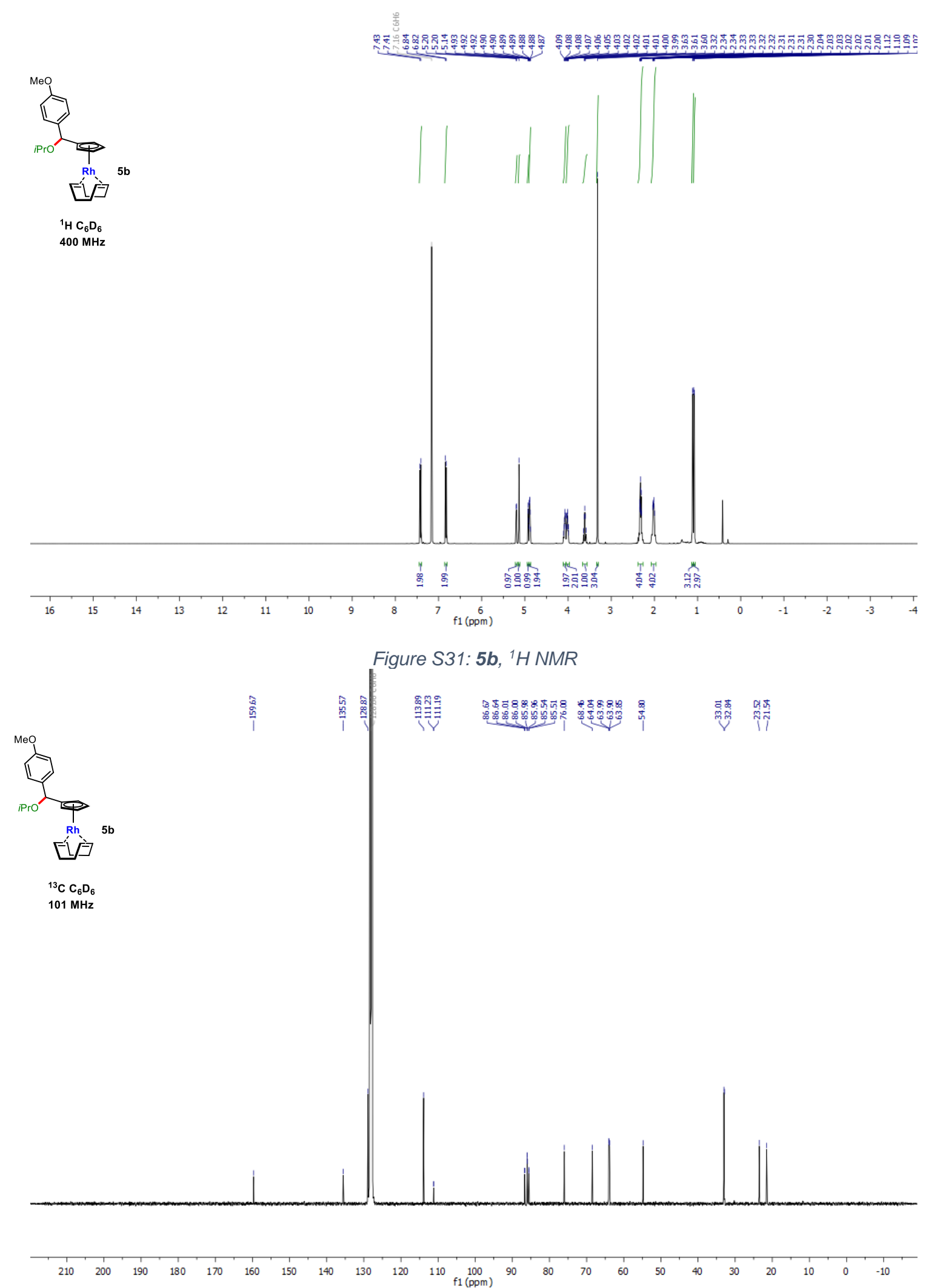

Figure S32: $5 b,{ }^{13} \mathrm{C} N M R$ 


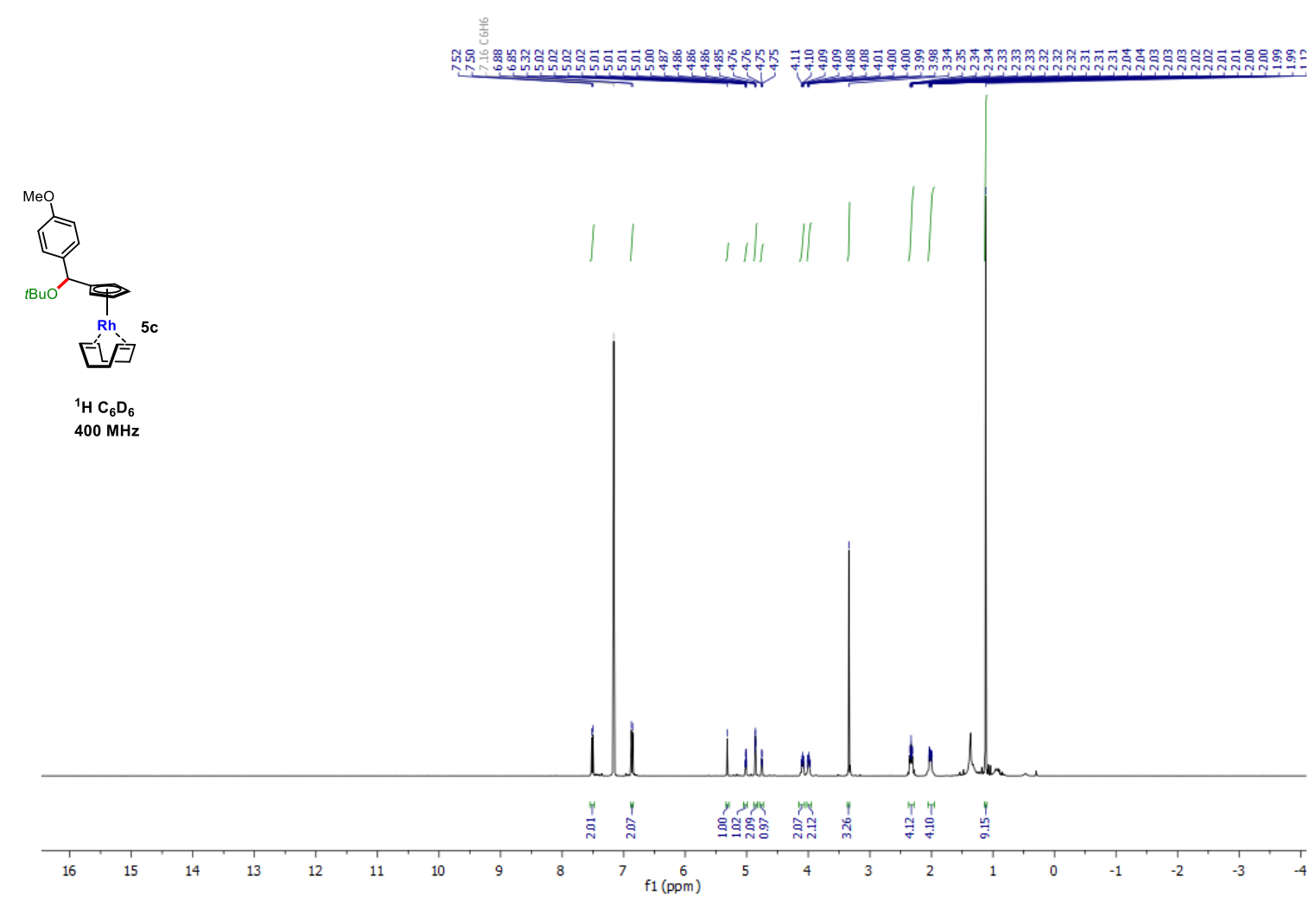

Figure S33: $5 c,{ }^{1} H$ NMR
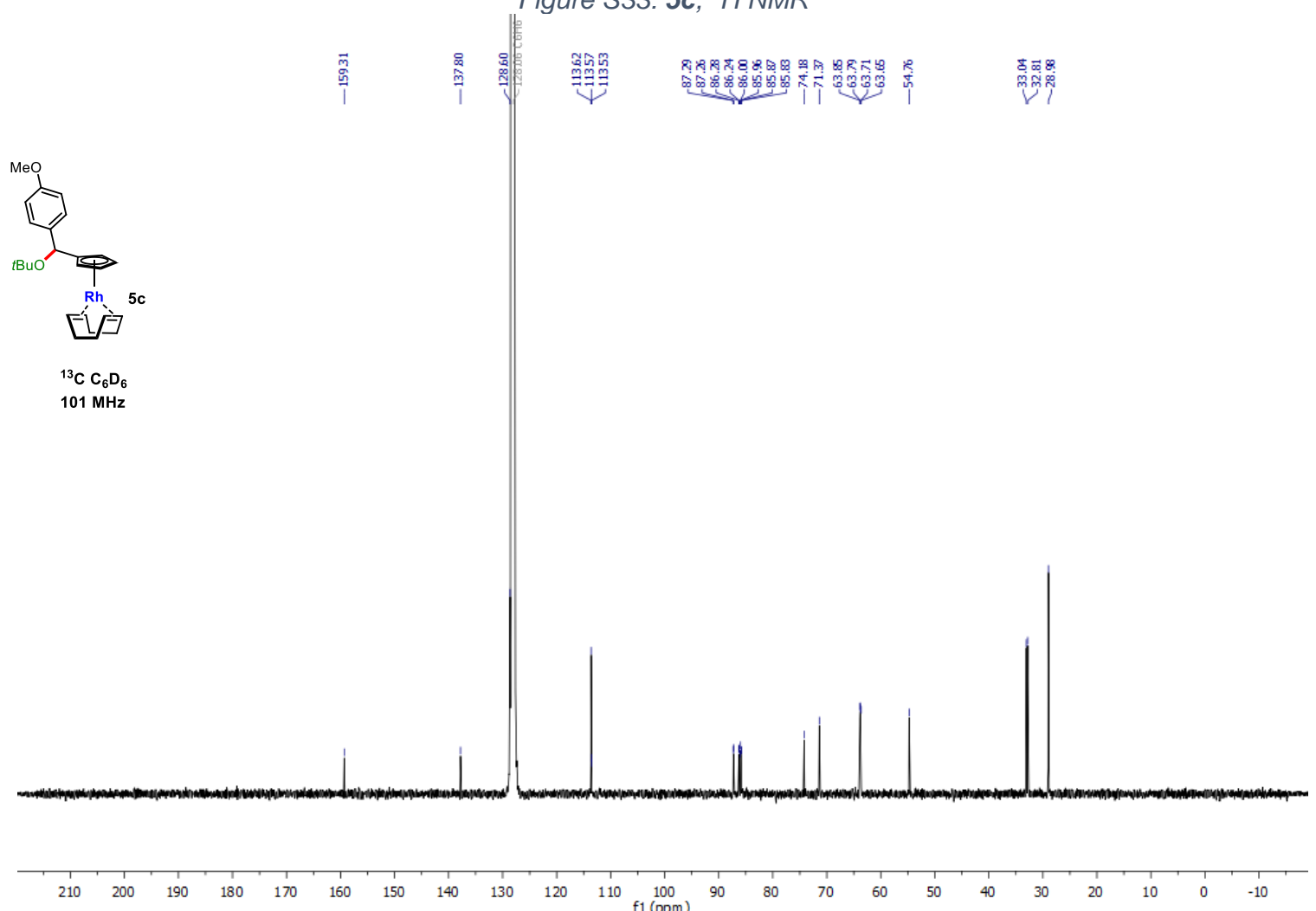

Figure S34: $5 c,{ }^{13} \mathrm{C}$ NMR 

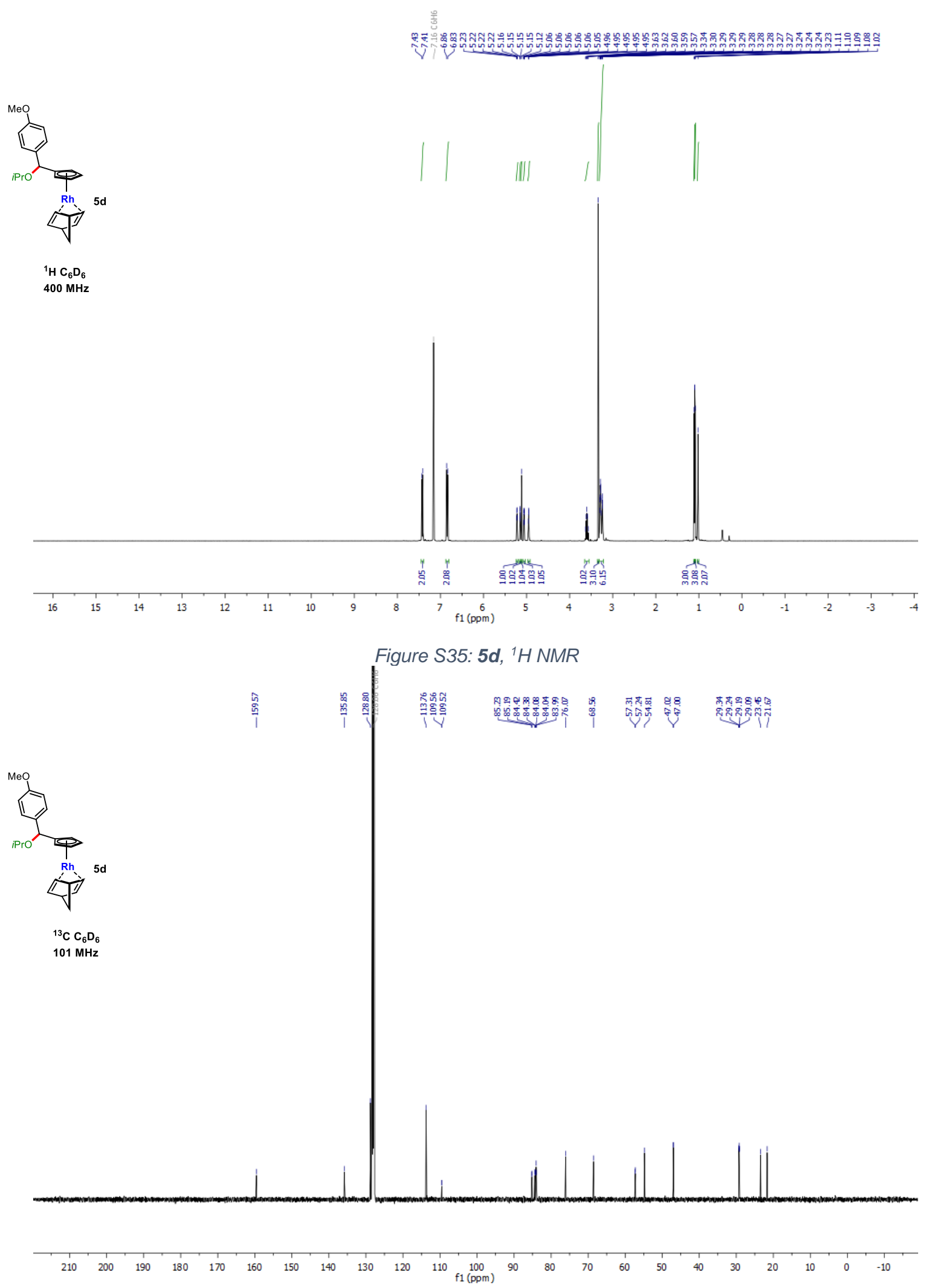

Figure S36: $5 d,{ }^{13} \mathrm{C}$ NMR 

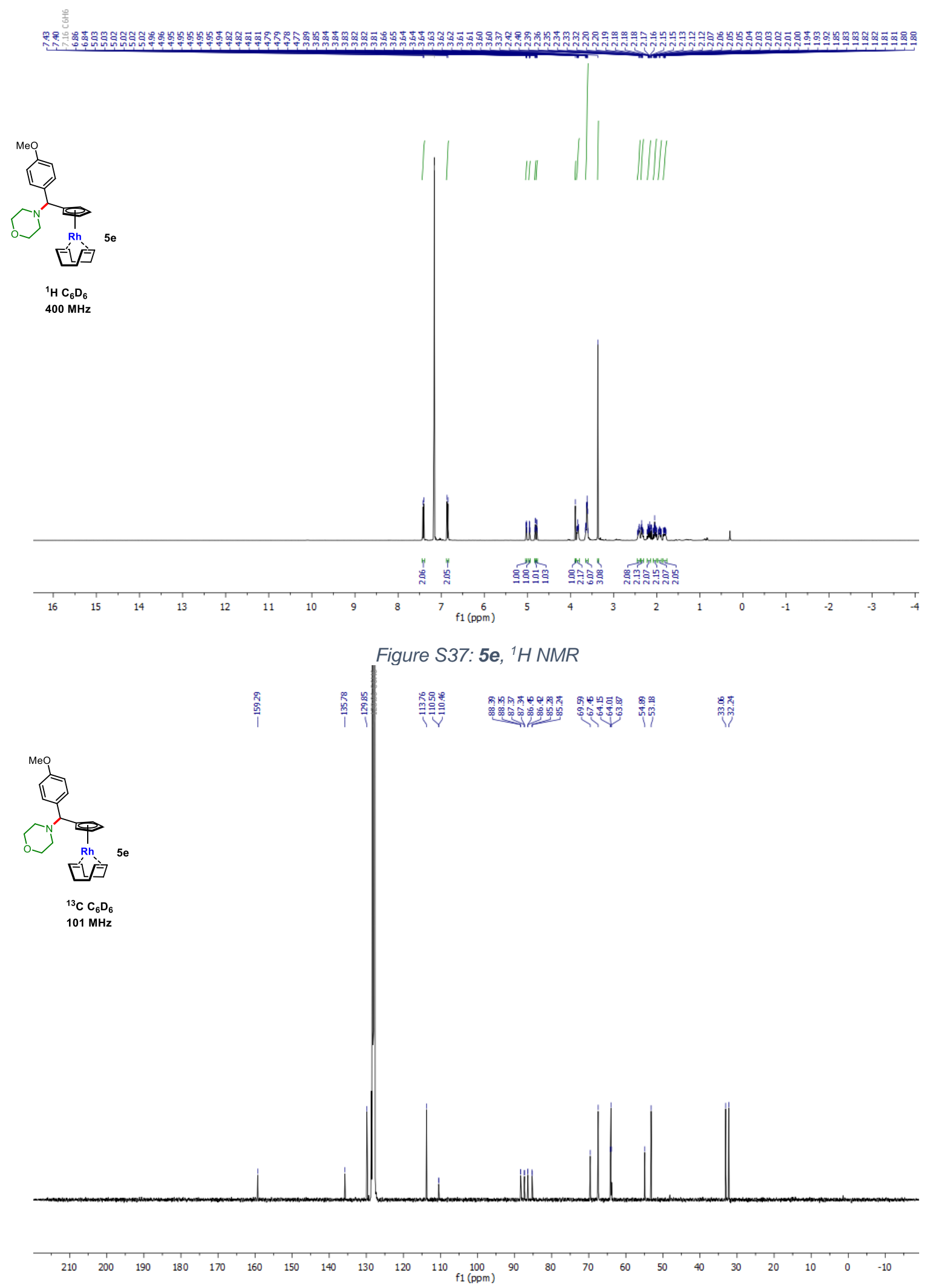

Figure S38: $5 \boldsymbol{e},{ }^{13} \mathrm{C}$ NMR 

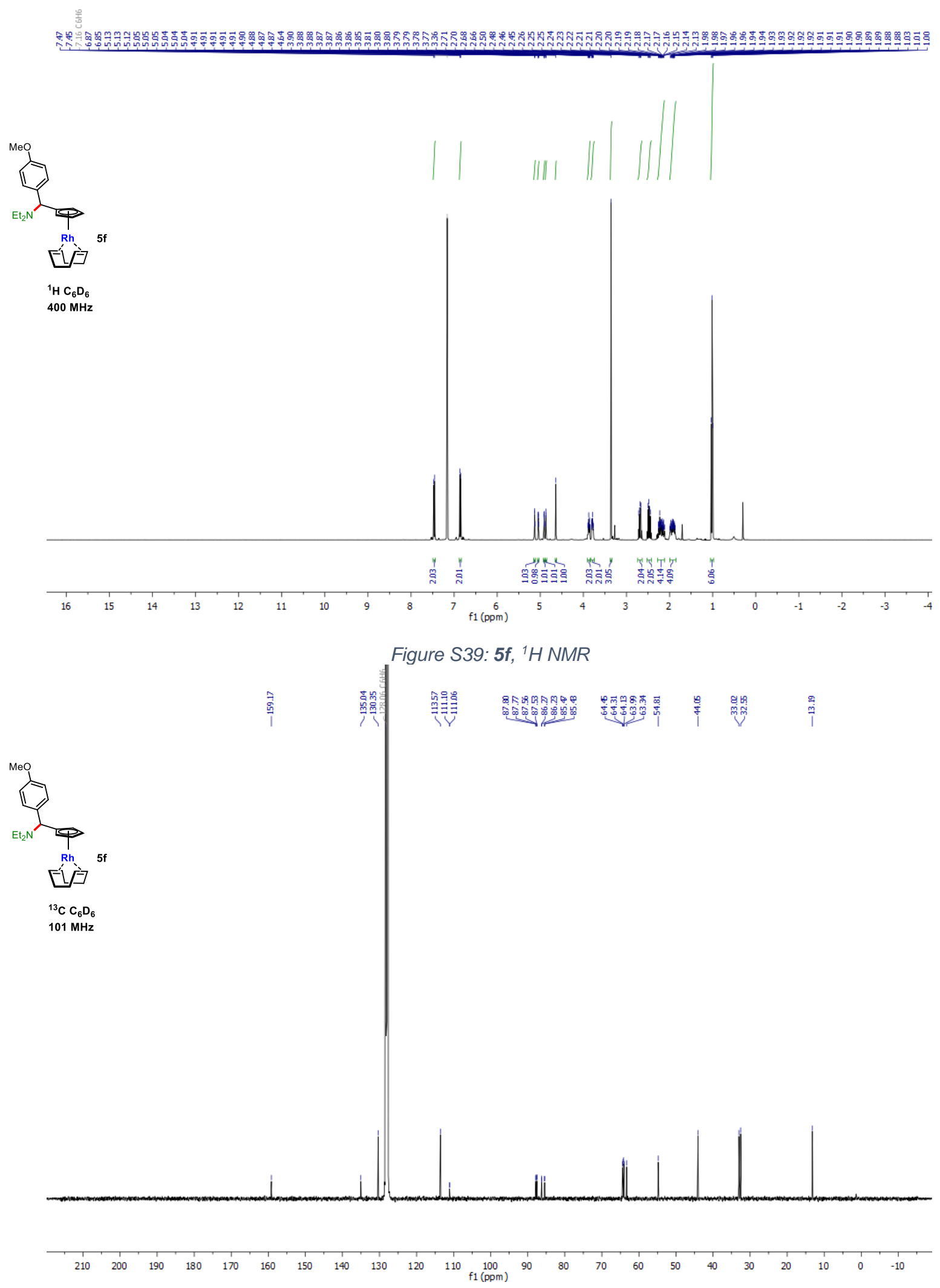

Figure S40: $5 f,{ }^{13} \mathrm{C}$ NMR 

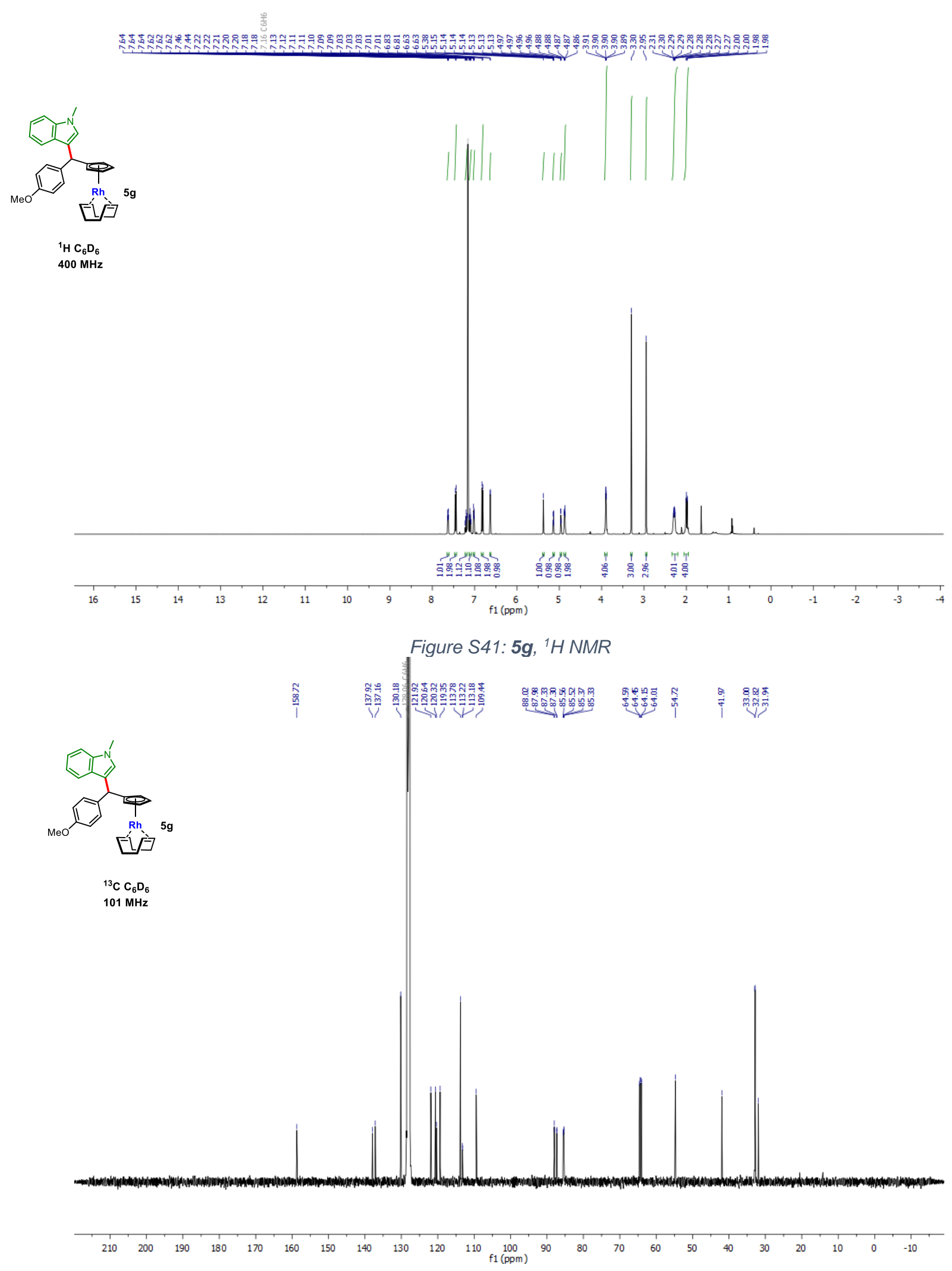

Figure S42: $5 g,{ }^{13} \mathrm{C}$ NMR 

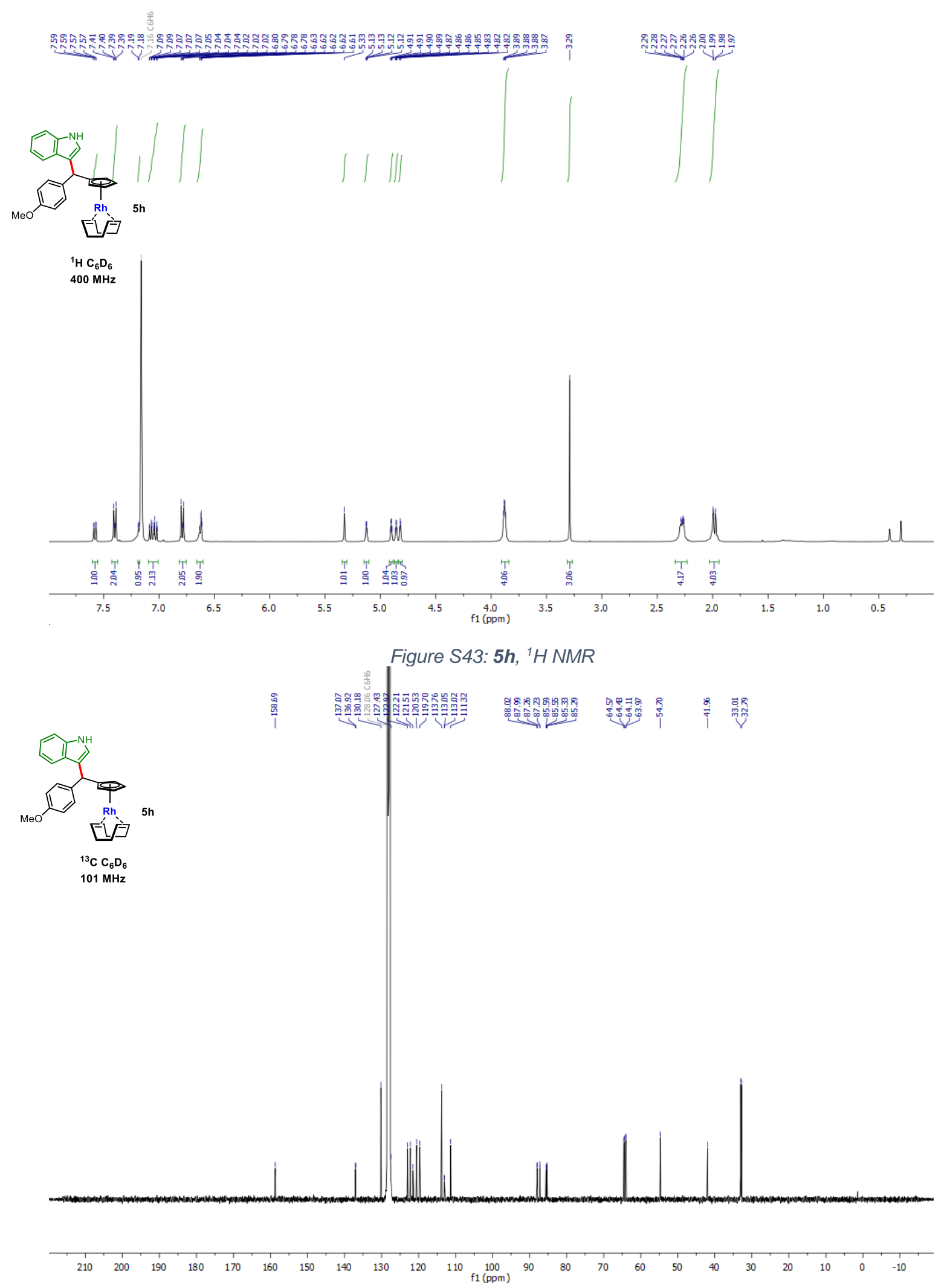

Figure S44: 5 h, ${ }^{13} \mathrm{C}$ NMR 

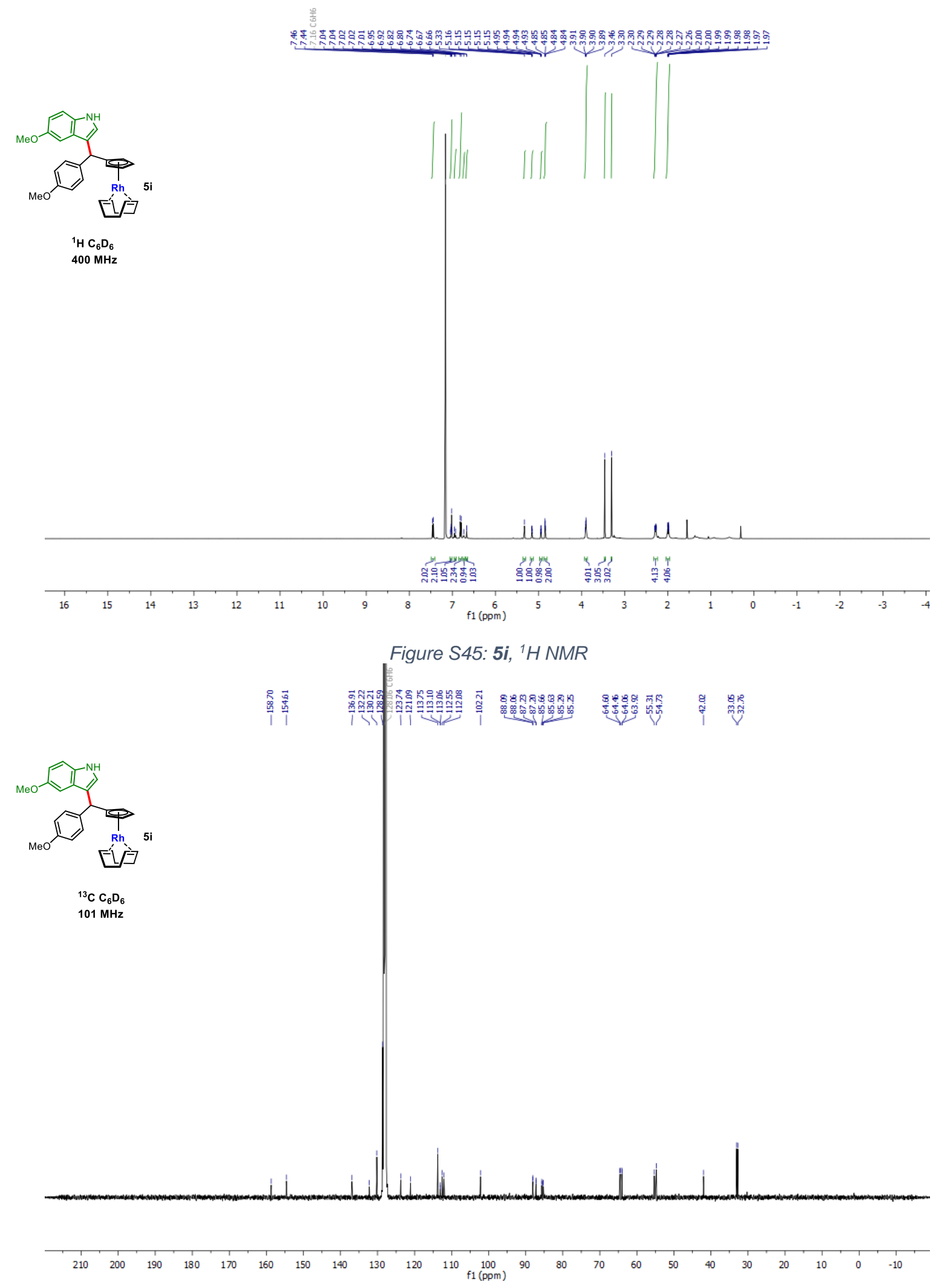

Figure S46: $5 i,{ }^{13} \mathrm{C}$ NMR 

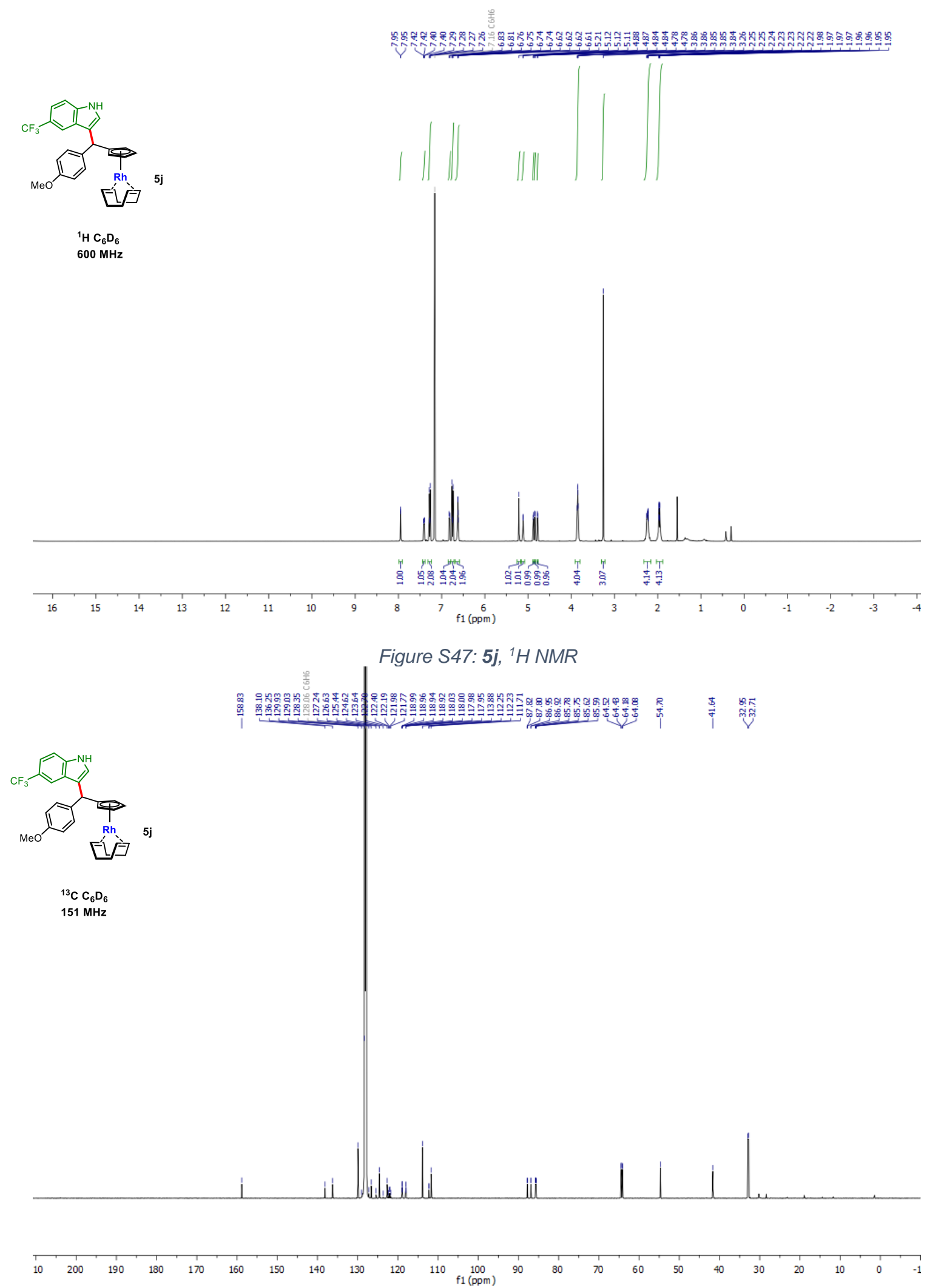

Figure S48: $5 j,{ }^{13} \mathrm{C} N M R$ 


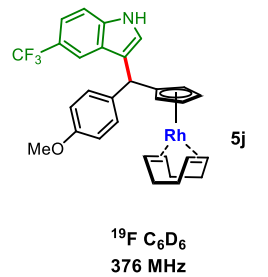

$376 \mathrm{MHz}$

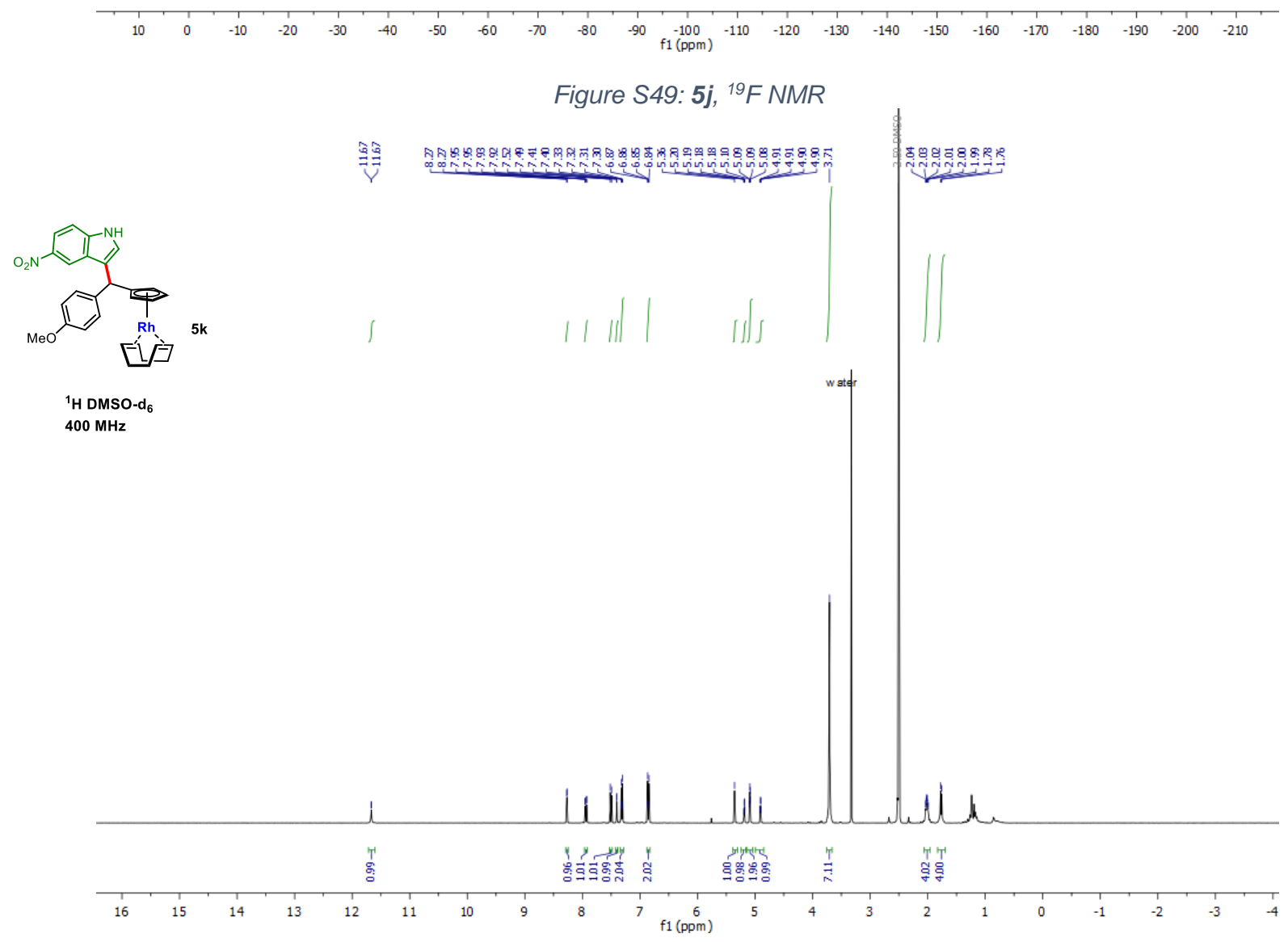

Figure S50: $5 k,{ }^{1} H$ NMR 


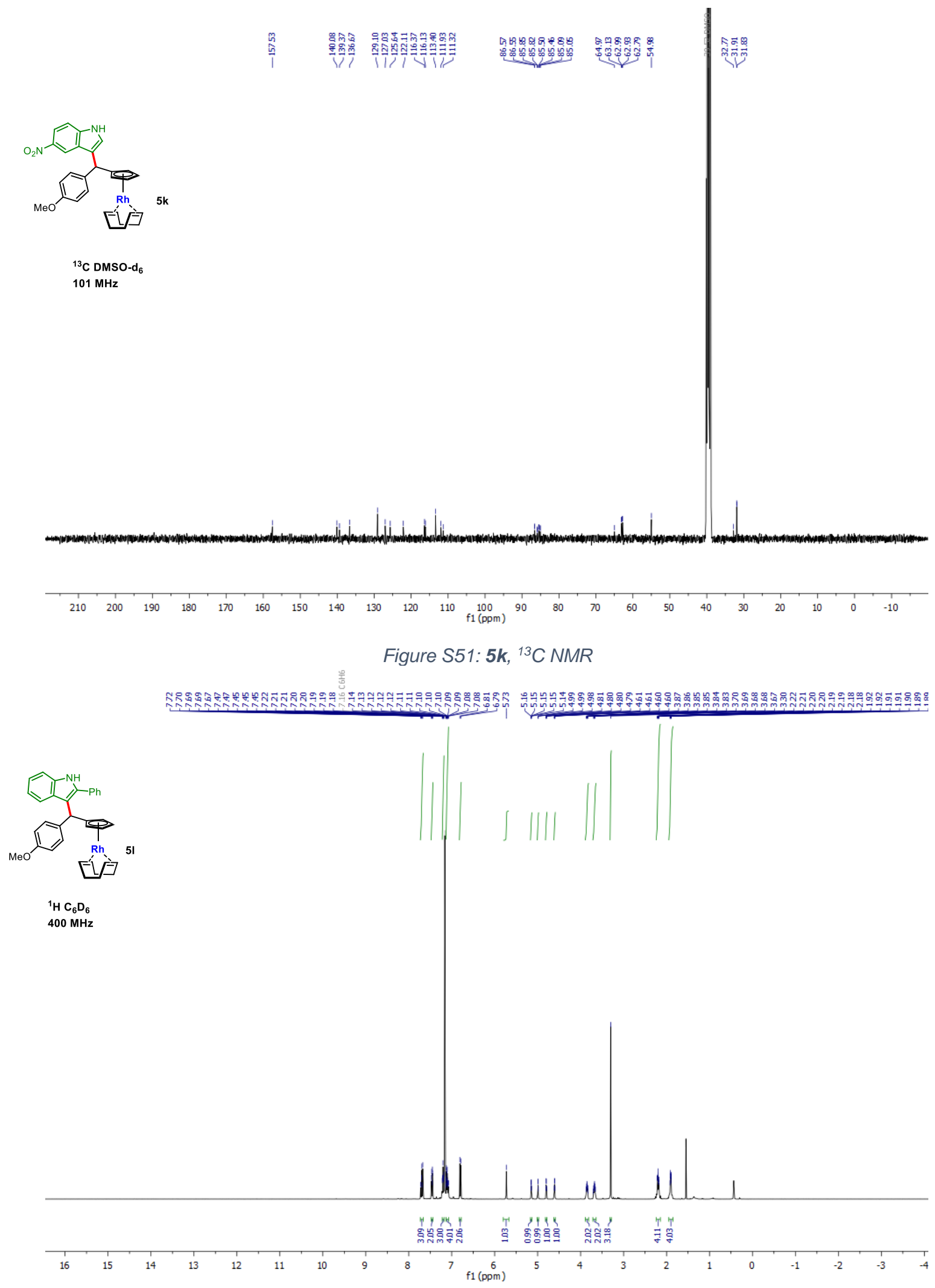

Figure S52: $51,{ }^{1} \mathrm{H} N \mathrm{NR}$ 


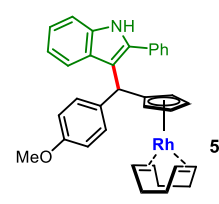

${ }^{13} \mathrm{C} \mathrm{C}_{6} \mathrm{D}_{6}$

$101 \mathrm{MHz}$

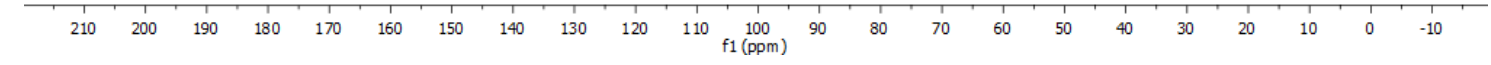

Figure S53: 5I, ${ }^{13} \mathrm{C}$ NMR

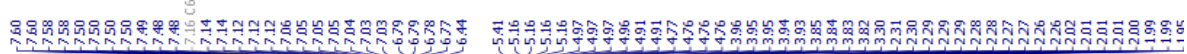
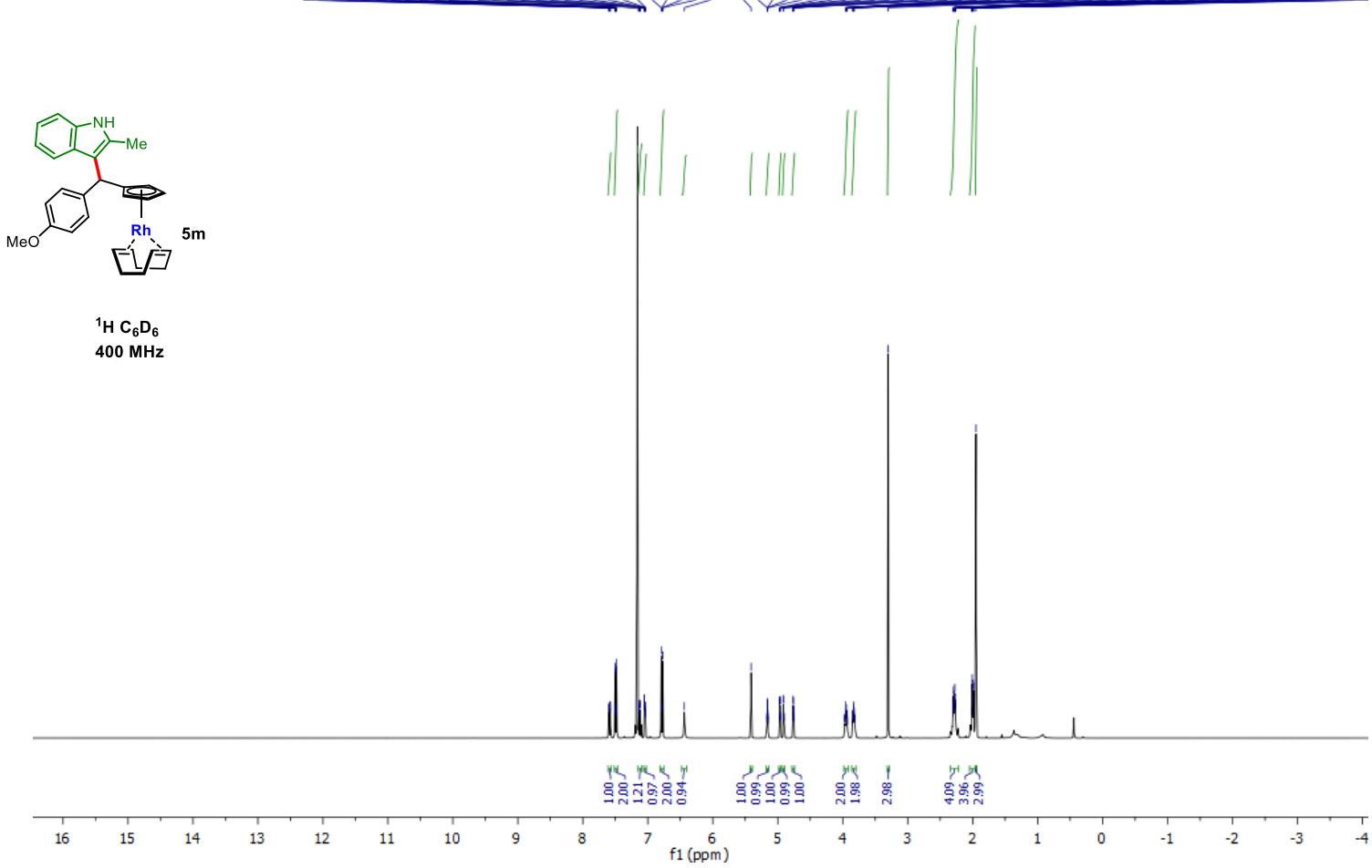

Figure S54: $5 m,{ }^{1} H$ NMR 

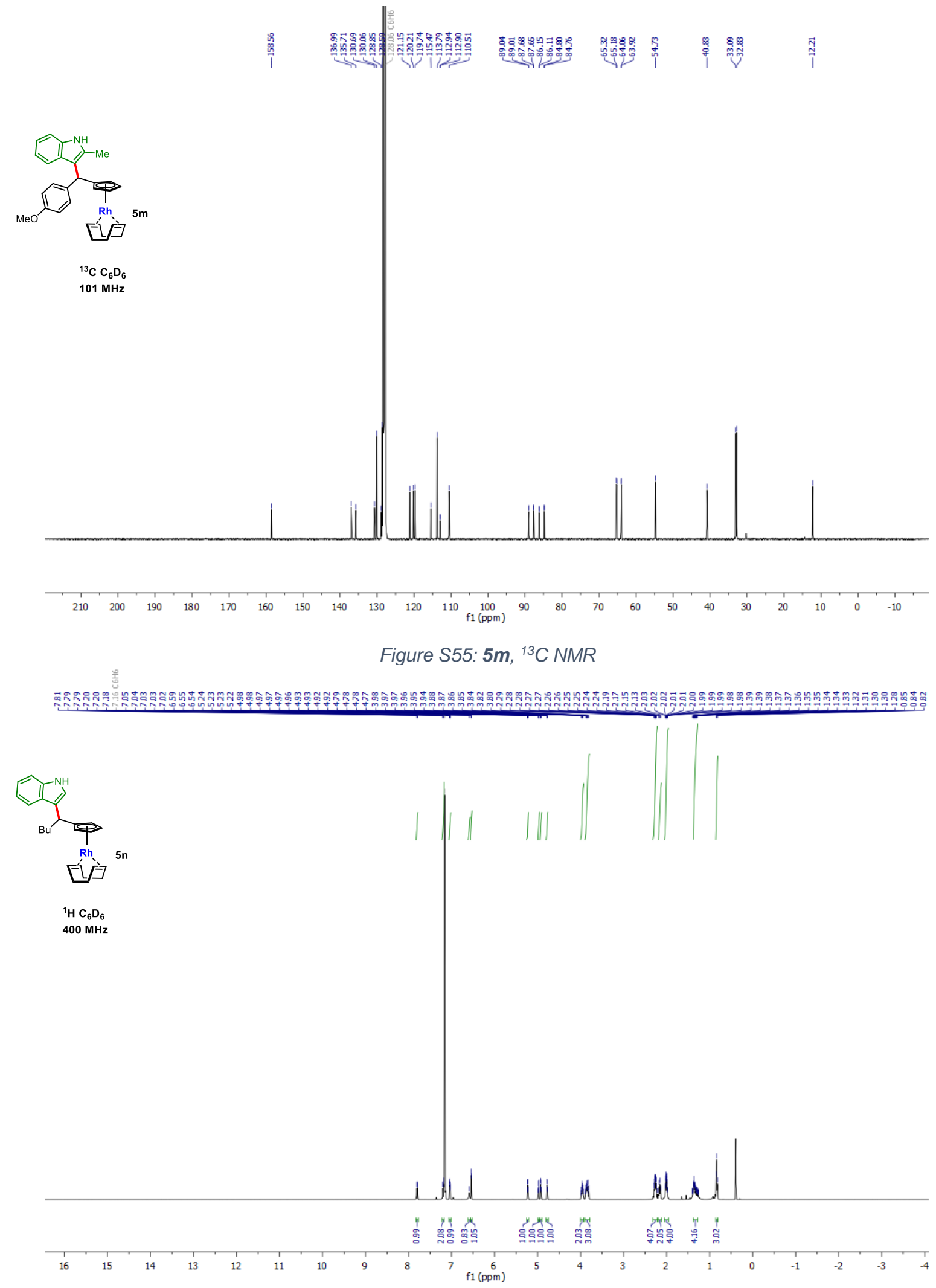

Figure S56: $5 n,{ }^{1} H$ NMR 

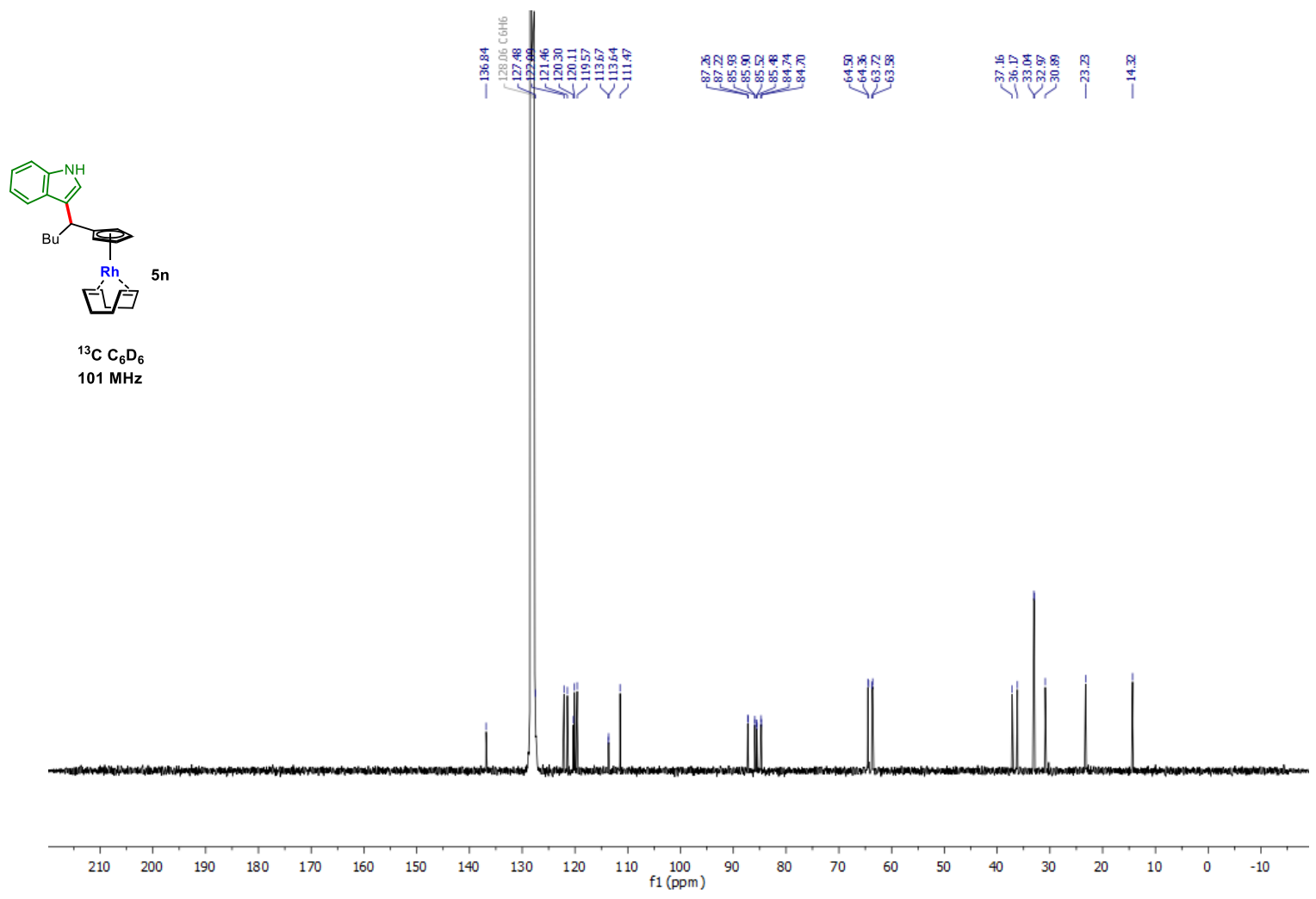

Figure S57: $5 n,{ }^{13} \mathrm{C}$ NMR

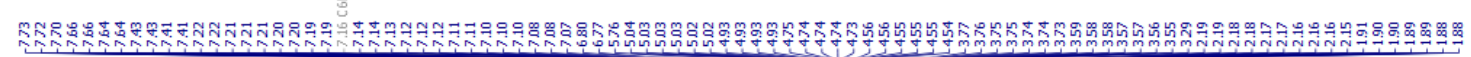
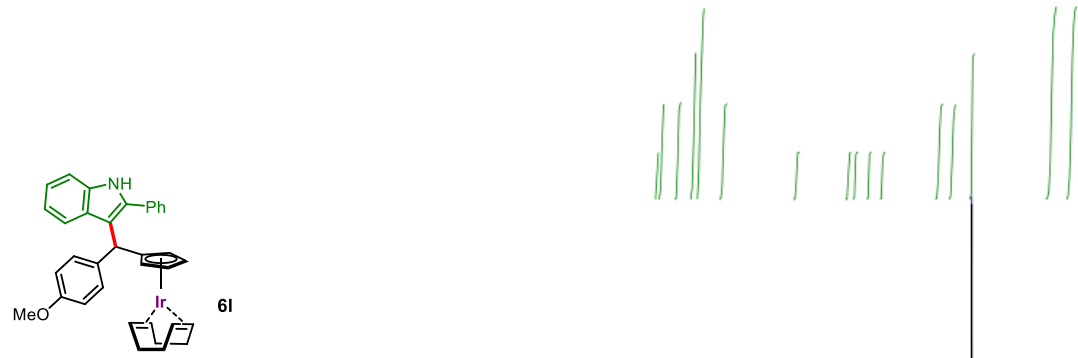

${ }^{1} \mathrm{HC}_{6} \mathrm{D}_{6}$

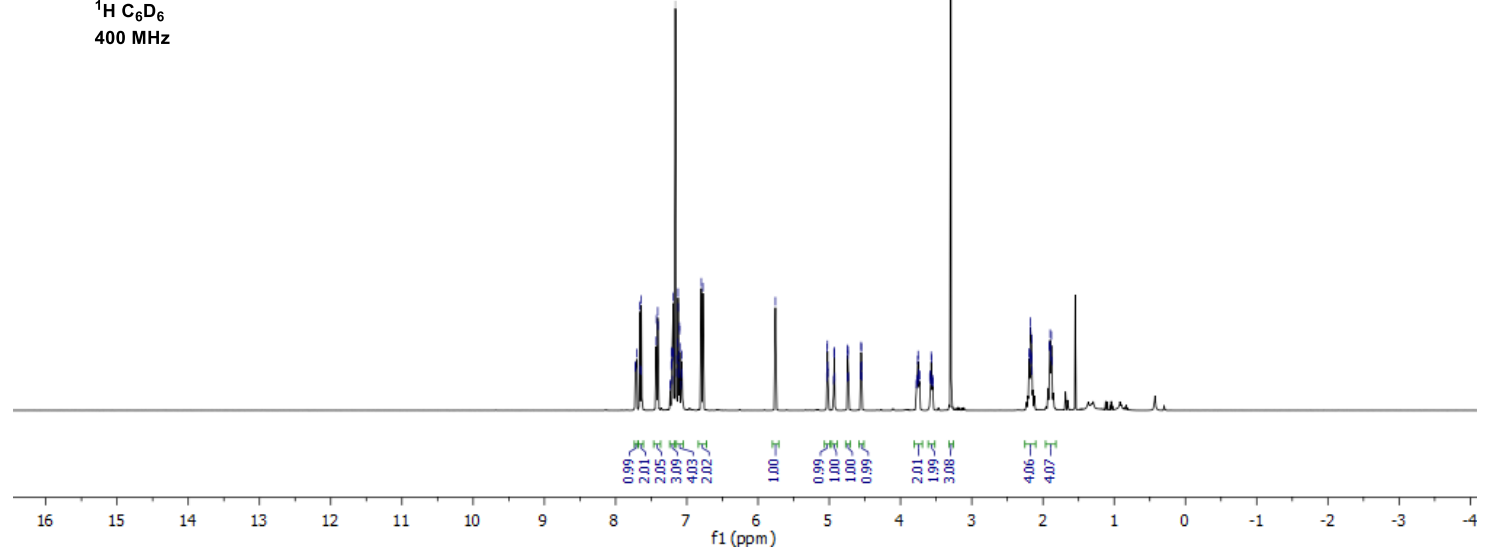

Figure S58: $61,{ }^{1} \mathrm{H} N \mathrm{MR}$ 

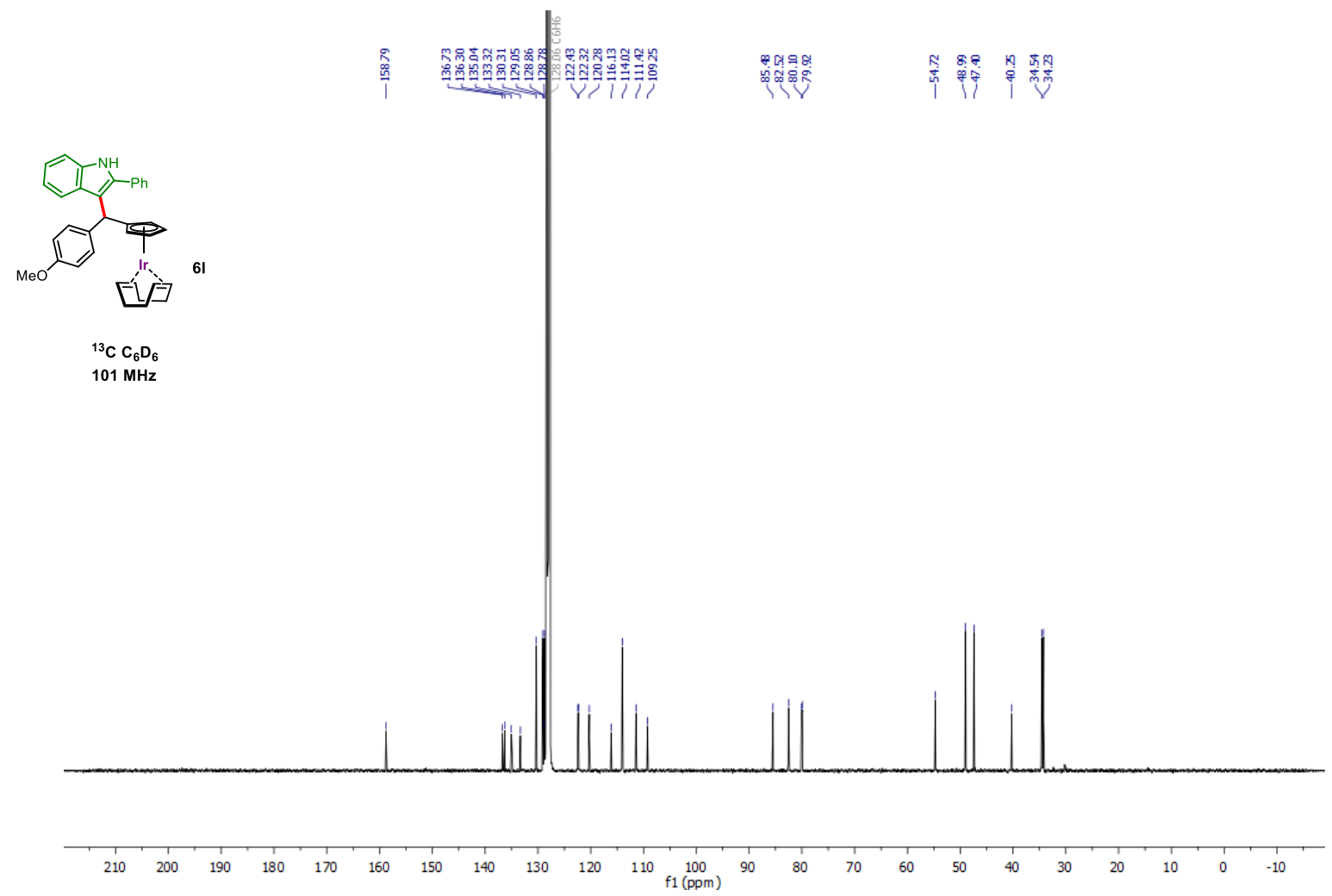

Figure S59: 6I, ${ }^{13} \mathrm{C}$ NMR

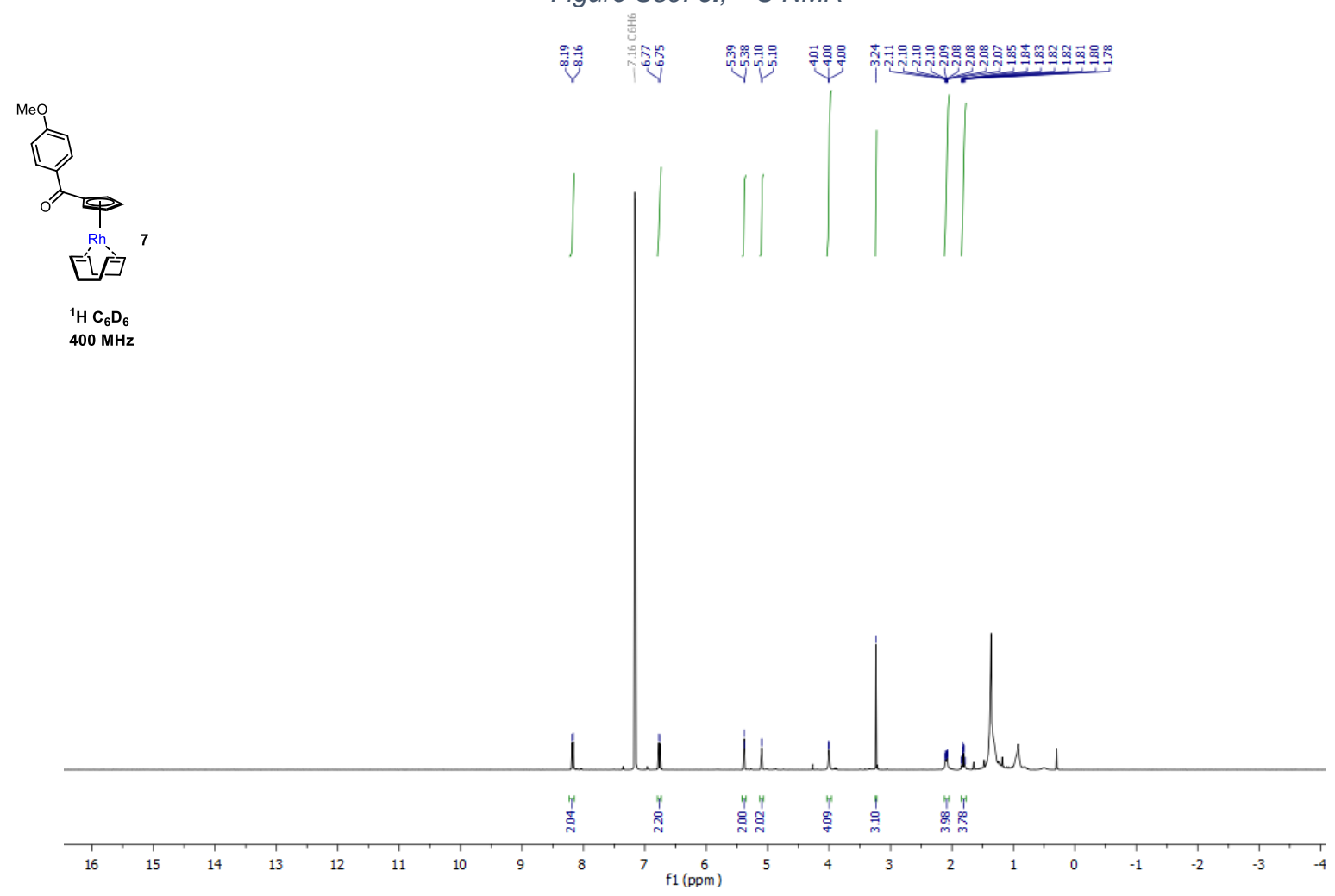

Figure S60: 7, ${ }^{1} \mathrm{H}$ NMR 

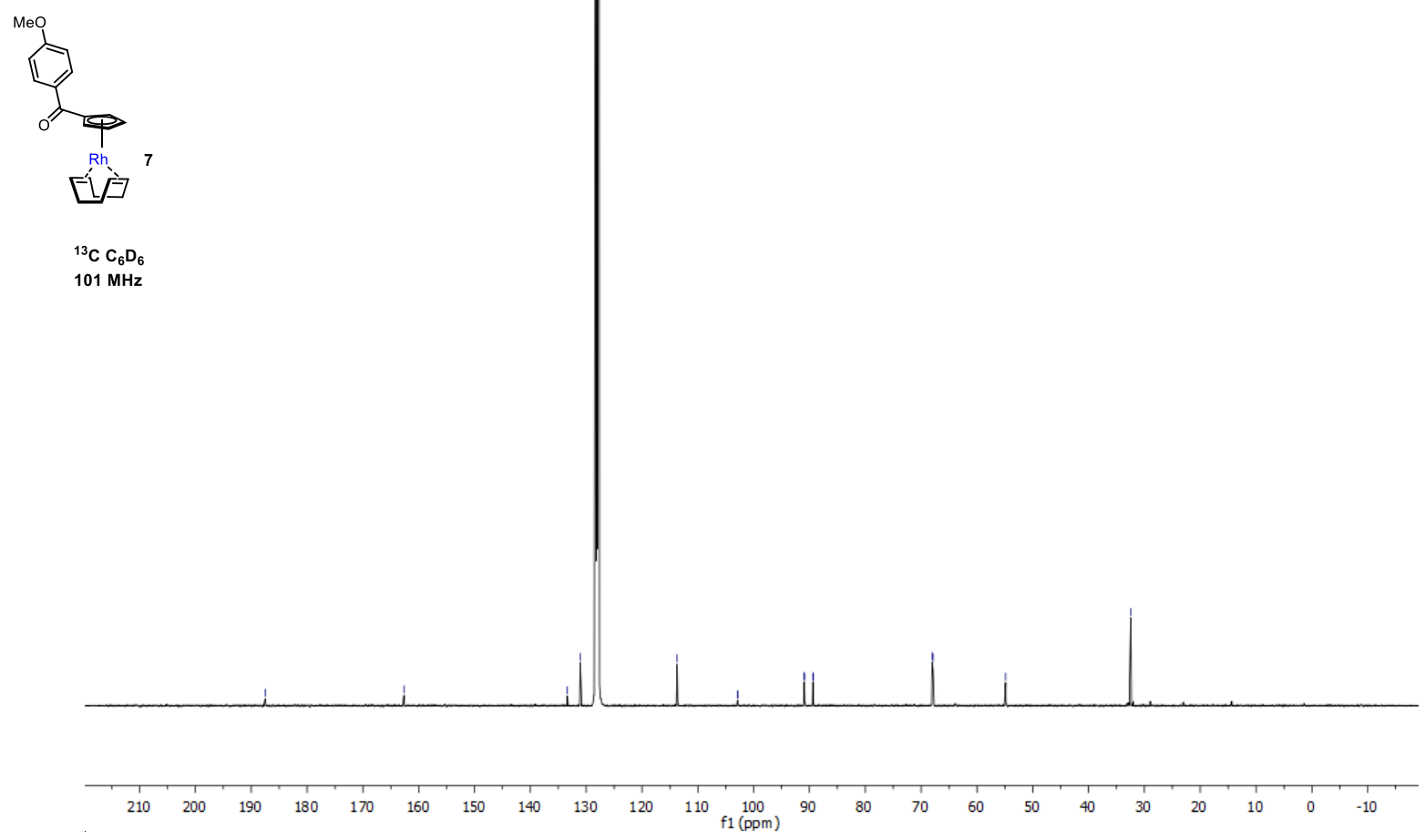

Figure S61: 7, ${ }^{13} \mathrm{C}$ NMR

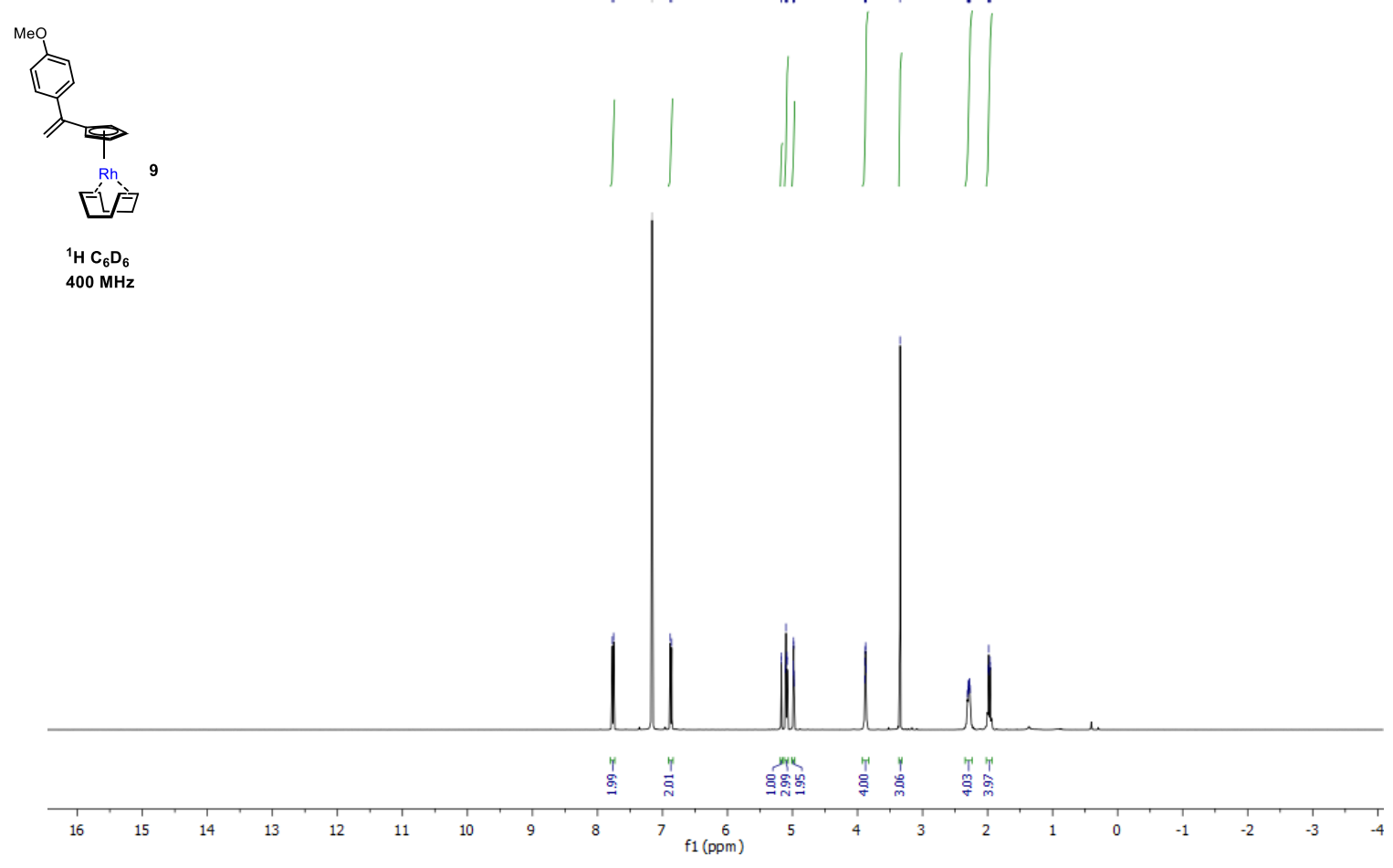

Figure S62: 9, ${ }^{1} \mathrm{H}$ NMR 


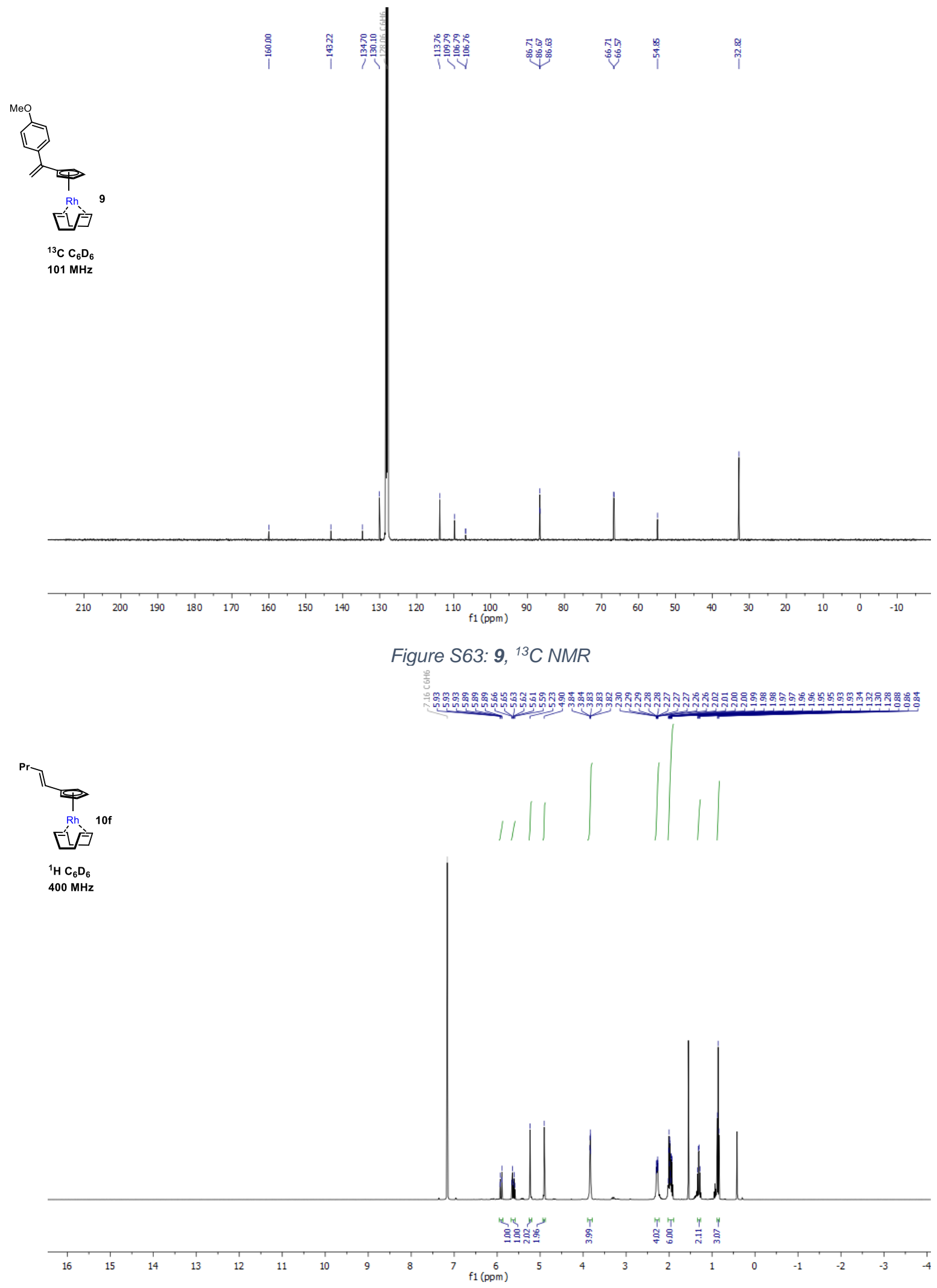

Figure S64: 10f, ${ }^{1} \mathrm{H}$ NMR 

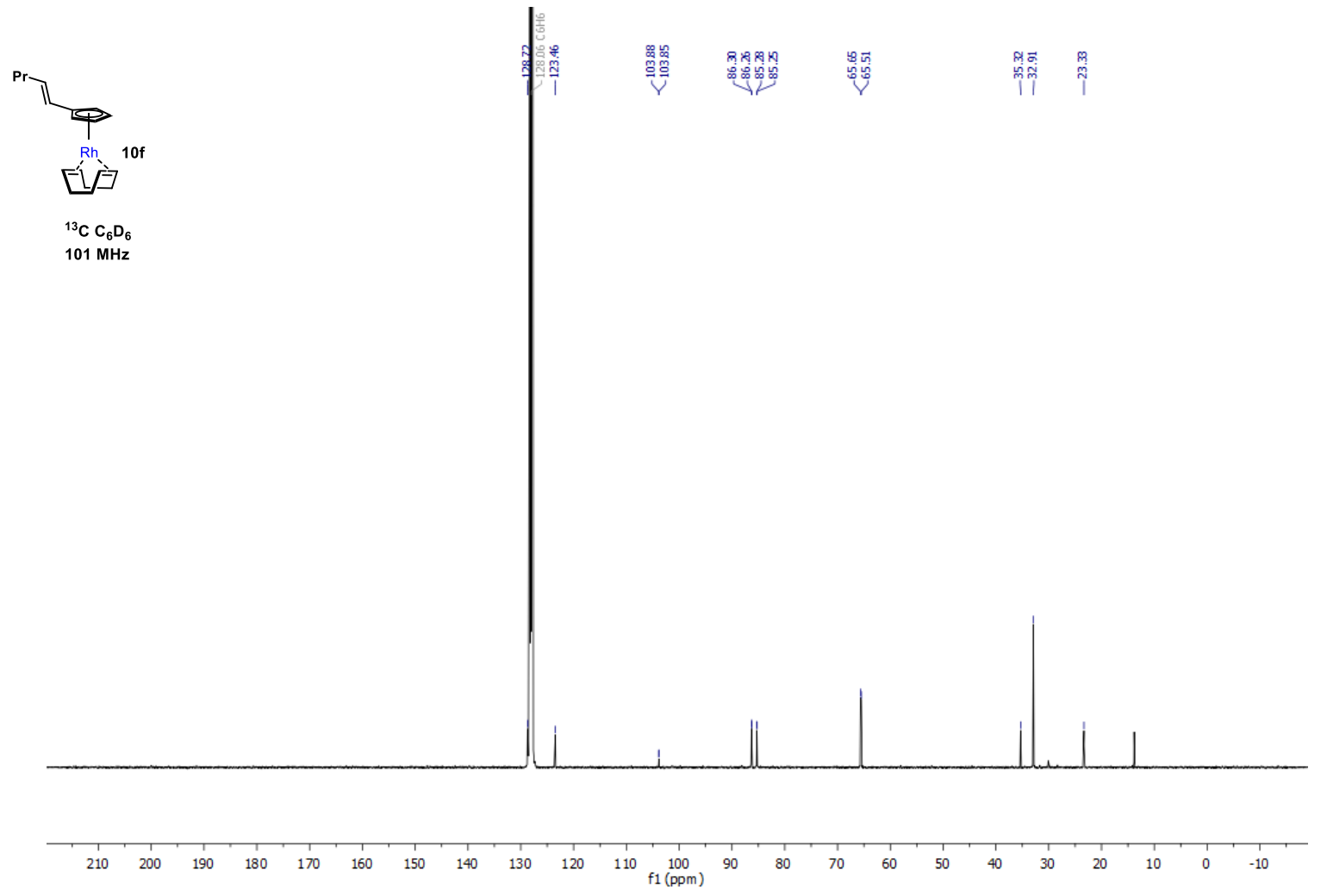

Figure S65: $10 f,{ }^{13} \mathrm{C}$ NMR

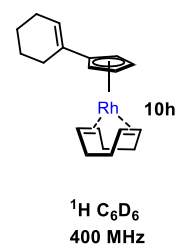

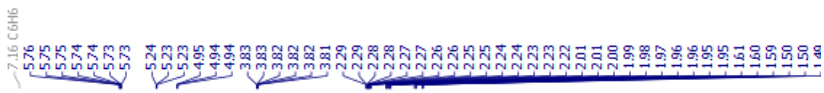

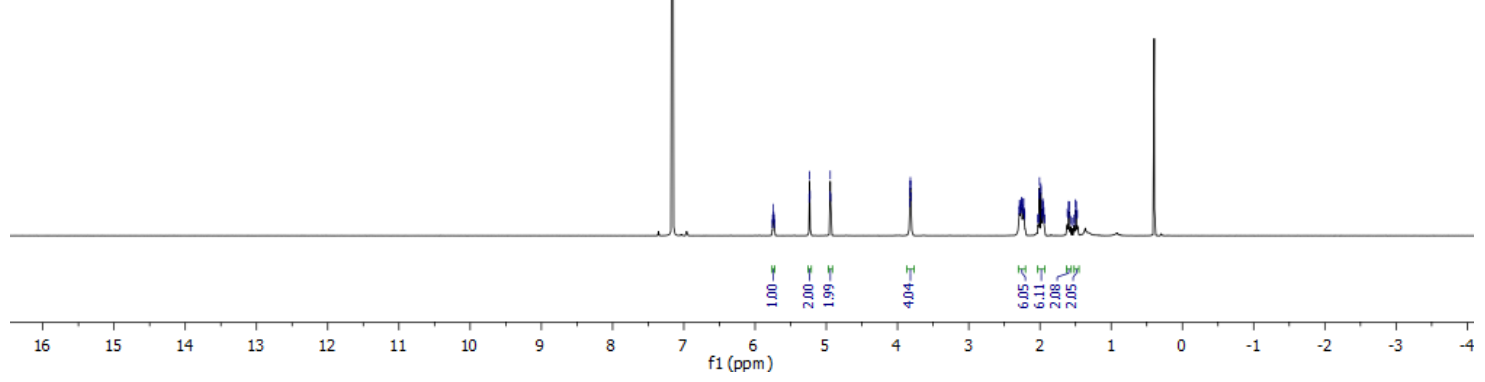

Figure S66: 10h, ${ }^{1} \mathrm{H}$ NMR 


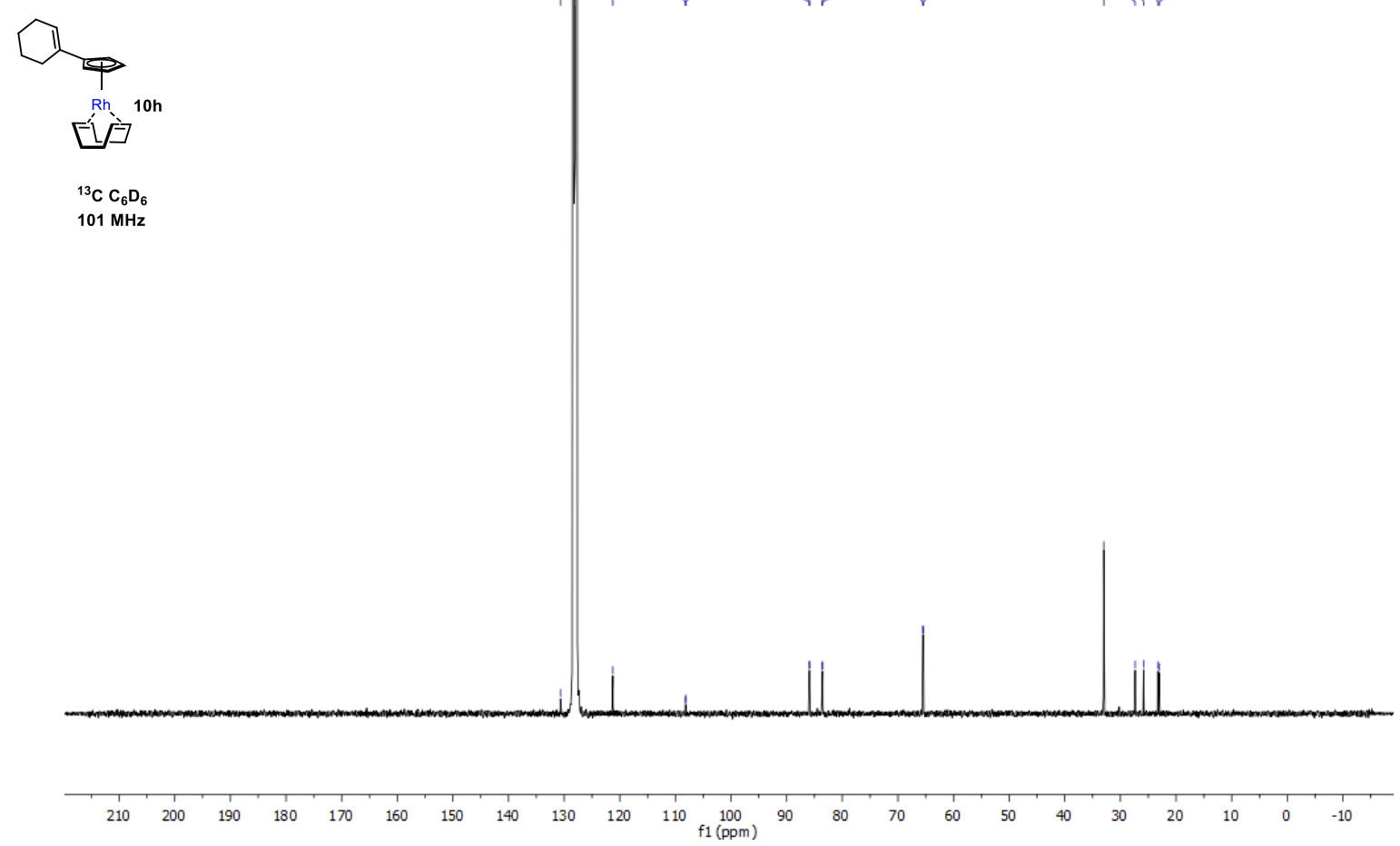

Figure S67: 10h, ${ }^{13} \mathrm{C} N \mathrm{NMR}$ 